Discussion Paper No. 884

\title{
BERTRAND COMPETITION \\ UNDER NETWORK EXTERNALITIES
}

\author{
Masaki Aoyagi
}

September 2013

The Institute of Social and Economic Research Osaka University

6-1 Mihogaoka, Ibaraki, Osaka 567-0047, Japan 


\title{
Bertrand Competition under Network Externalities
}

\author{
Masaki Aoyagi* \\ Osaka University
}

September 6, 2013

\begin{abstract}
Two sellers engage in price competition to attract buyers located on a network. The value of the good of either seller to any buyer depends on the number of neighbors on the network who consume the same good. For a generic specification of consumption externalities, we show that an equilibrium price equals the marginal cost if and only if the buyer network is complete or cyclic. When the externalities are approximately linear in the size of consumption, we identify the class of networks in which one of the sellers monopolizes the market, or the two sellers segment the market.

Key words: graphs, networks, externalities, Bertrand, divide and conquer, discriminatory pricing, monopolization, segmentation, two-sided market.

Journal of Economic Literature Classification Numbers: C72, D82.
\end{abstract}

\section{Introduction}

Goods have network externalities when their value to each consumer depends on the consumption decisions of other consumers. The externalities may derive from physical connection to consumers adopting the same good as in the case of telecommunication devices, from provision of complementary goods as in the case of operating systems and softwares for computers, or from pure psychological factors as in the case of consumption bandwagon. Despite their importance in reality, we only have limited understanding of network externalities particularly when those goods are supplied competitively. The objective of this paper is to study price competition in the presence of consumption externalities represented by a buyer network.

${ }^{*}$ ISER, Osaka University, 6-1 Mihogaoka, Ibaraki, Osaka 567-0047, Japan. 
Specifically, we formulate a model of price competition under local network externalities by supposing that two sellers compete for a network of buyers who experience externalities when their neighbors in the network consume the same good.

A more detailed description of our model is as follows: Two sellers each sell goods that are incompatible with each other. Consumers of either good experience larger positive externalities when more of his neighbors in the network consume the same good. In stage 1, the two sellers post prices simultaneously. The prices can be perfectly discriminatory and can be negative. Upon publicly observing the price vectors posted by both sellers, the buyers in stage 2 simultaneously decide which good to buy or not to buy. The sellers have no cost of serving the market, and their payoffs simply equal the sum of prices offered to the buyers who choose to buy their goods.

In this framework, we find that the equilibrium outcome of price competition subtly depends on the network structure. Our first observation concerns the validity of marginal cost pricing. When no network externalities are present, it is clear that the unique subgame perfect equilibrium of this game has both sellers offer zero to all buyers. In the presence of externalities, however, we show that the marginal cost pricing is consistent with equilibrium only if either the externalities are linear (in the number of neighbors consuming the same good), or the network is either a complete graph or a cycle. ${ }^{1}$ In any other network, if the externalities generic, there exists no equilibrium in which either seller captures the entire market by offering the same price to all buyers. This is so even in networks where all buyers have symmetric locations. Given this surprising result, we attempt to identify equilibrium prices under non-linear externalities.

Positive identification of equilibrium prices is possible when the externalities are close to linear and when the network satisfies certain properties as follows. First, we consider bipartite networks. A network is bipartite if the set of buyers can be divided into two subsets such that for every buyer in either subset, all his neighbors belong to the other subset. This is an important class of networks given that it corresponds to a two-sided market that has received much attention in the literature as discussed in the next section. We show that in a bipartite network, there exists an equilibrium in which one of the sellers captures the entire market (i.e., buyers on both sides) by charging positive prices to all buyers on one side while subsidizing all buyers on the

\footnotetext{
${ }^{1} \mathrm{~A}$ graph is complete if any pair of buyers are neighbors. The linear externalities in particular imply that the value of the good is zero to a buyer when none of his neighbors consumes it.
} 
other side.

Next, we identify the class of networks for which market segmentation takes place in equilibrium. We say that a network is separable if the buyer set can be divided into two subsets such that every buyer in each subset has at least as many neighbors in the same subset as in the other subset, and some buyer in each subset has strictly more neighbors in the same subset than in the other subset. In a separable network, we show that market segmentation takes place in equilibrium with each seller making strictly positive profits.

The paper is organized as follows: After discussing the related literature in the next section, we formulate a model of price competition in Section 3. Section 4 considers the subgame played by the buyers that follows the public observation of prices posted by both sellers. The critical observations there are that this simultaneousmove game is one of strategic complementarity, and hence that there exist maximal and minimal Nash equilibria in each subgame. We present an algorithm to obtain these extreme equilibria and use them in our construction of a subgame perfect equilibrium of the entire game. We begin the analysis of a subgame perfect equilibrium in Section 5 and identify lower bounds on the sellers' payoff in such equilibrium. Section 6 examines the validity of marginal cost pricing in equilibrium. With the definition of approximate linearity, Section 7 discusses equilibrium in a bipartite network, which corresponds to a model of two-sided markets. Equilibrium market segmentation in separable networks is discussed in Section 8. We conclude in Section 9. All the proofs are collected in the Appendix. The Appendix also contains an analysis of the game when the buyers coordinate their actions by playing a strong Nash equilibrium in the stage 2 subgame.

\section{Related Literature}

Dybvig and Spatt (1983) are the first to theoretically study the provision of goods with network externalities. ${ }^{2}$ The problems of a single supplier of a good with network externalities are subsequently studied by Cabral et al. (1999), Park (2004), Sekiguchi (2009), Ochs and Park (2010), Aoyagi (2013), among others. These papers focus on such issues as the construction of efficient or revenue maximizing adoption schemes under complete and incomplete information, intertemporal patterns of adoption decisions, as well as the validity of introductory pricing.

\footnotetext{
${ }^{2}$ Rohlfs (1974) provides a very early discussion of network externalities.
} 
Segal (2003), Winter (2004) and Bernstein and Winter (2012) study a closely related problem of contracting under externalities in which a single principal offers a contract to the set of agents whose participation decisions create externalities to other agents. They discuss the so-called divide-and-conquer strategy used by the principal: According to the strategy, the principal approaches agents one by one in some order. The contract offered to the first agent induces him to participate even if all other agents abstain. The contract offered to the second agent induces him to participate if all but the first agent abstain, and so on. In our analysis of an equilibrium, we use exactly the same argument: Given some price profile, we examine if it is profitable for either seller to approach the buyers one by one in some order with prices that induce them to choose the buyer provided that all their predecessors do the same. We note that the argument is essentially that of iterative elimination of strictly dominated strategies, and show that it can be used very effectively to examine if the given price profile is part of an equilibrium.

Competition between suppliers of goods with network externalities was first formulated by Katz and Shapiro (1985), and subsequently studied by Sundararajan (2003), Ambrus and Argenziano (2009), Bernaji and Dutta (2009), and Jullien (2011). These models are often couched in terms of two-sided markets, where the sellers are providers of platforms who offer a marketplace for agents on two sides such as sellers and buyers of some good. In such models, the utility of an agent on one side is an increasing function of the number of participants from the other side. ${ }^{3}$ Ambrus and Argenziano (2009) analyze Bertrand competition between platforms in a two-sided market. Jullien (2011) applies the divide-and-conquer argument to his analysis of multi-sided markets, and derives a bound on the platforms' payoffs when they engage in Stackelberg price competition. Both Ambrus and Argenziano (2009) and Jullien (2011) formulate externalities differently from the present paper, and impose some non-trivial restrictions on the agents' strategies. Although these restrictions may appear natural under some price profiles, their full implications are not immediately clear. In contrast, our analysis of a subgame perfect equilibrium imposes no restriction on the buyers' strategies.

To the best of our knowledge, Banerji and Dutta (2009) are the only other paper that introduces graph structure into a model of price competition under network externalities. They identify conditions under which price competition leads to monopolization and market segmentation. They assume, however, that each seller sets

\footnotetext{
${ }^{3}$ See Armstrong (1998), and Laffont et al. (1998a,b).
} 
the same price for all buyers and also place restrictions on the buyers' strategies. Because of these differences in assumptions, their conclusions are difficult to compare with ours.

\section{Model}

Two sellers $A$ and $B$ compete for the set $I=\{1, \ldots, N\}$ of $N \geq 3$ buyers. Consumption of either seller's good generates externalities to the buyers according to a buyer network. Formally, a buyer network is represented by a simple undirected graph $G$ whose nodes correspond to the buyers, and consumption externalities exist between buyers $i$ and $j$ if they are adjacent in the sense that there is a link between $i$ and $j$. When buyer $j$ is adjacent to buyer $i$, we also say that $j$ is $i$ 's neighbor.

The buyer network $G$ is connected in the sense that for any pair of buyers $i$ and $j$, there exists a path from $i$ to $j$. That is, there exist buyers $i_{1}, i_{2}, \ldots, i_{m}$, such that $i_{1}$ is adjacent to $i, i_{2}$ is adjacent to $i_{1}, \ldots$, and $i_{m}$ is adjacent to $j$. For any buyer $i$ in network $G$, denote by $N_{i}(G)$ (or simply $N_{i}$ ) the set of $i$ 's neighbors in $G$. The degree $d_{i}(G)=\left|N_{i}(G)\right|$ of buyer $i$ in network $G$ is the number of $i$ 's neighbors. Define also $M$ to be the number of links in $G$. Since each link counts twice when aggregating the number of degrees in $G$, we have $M=\frac{1}{2} \sum_{i \in I} d_{i}$.

For $r=2, \ldots, N-1$, the network $G$ is $r$-regular if all buyers have the same degree $r$, and regular if it is $r$-regular for some $r$. $G$ is cyclic if it is connected and 2-regular, and complete if it is $(N-1)$-regular, or equivalently, every pair of buyers are adjacent to each other. For any non-empty subset $J \subset I$ of buyers, denote by $G[J]$ the subnetwork induced from $G$ : The set of nodes in $G[J]$ is $J$, and $G[J]$ has a link between $i \in J$ and $j \in J$ if and only if $i$ and $j$ are adjacent in the original network $G$.

The value of either seller's good to any buyer $i$ is determined by the number of neighbors of $i$ who consume the same good. We denote by $v^{n}$ the value of either good to any consumer when $n$ of his neighbors consume the same good. In particular, $v^{0}$ denotes the stand-alone value, or the value to any buyer of either good when none of his neighbors consumes the same good. The value does not depend on the identity of a buyer or the identity of the seller who supplies the good. The consumption externalities are non-negative in the sense that $0 \leq v^{0} \leq v^{1} \leq \cdots \leq v^{N-1}$.

Each seller produces his good at no fixed cost and a constant marginal cost. For simplicity, assume that the marginal costs also equals zero. Let $p_{i}$ and $q_{i}$ denote 
the prices offered to buyer $i$ by seller $A$ and seller $B$, respectively. The sellers can perfectly price discriminate the buyers. They simultaneously quote price vectors $p=\left(p_{i}\right)_{i \in I} \in \boldsymbol{R}^{N}$ and $q=\left(q_{i}\right)_{i \in I} \in \boldsymbol{R}^{N}$. The buyers publicly observe $(p, q)$, and then simultaneously decide whether to buy from either seller, or not buy. Buyer $i$ 's action $x_{i}$ is hence an element of the set $\{A, B, \emptyset\}$, where $\emptyset$ represents no purchase. Each seller's strategy is hence an element of $\boldsymbol{R}^{N}$, whereas buyer $i$ 's strategy $\sigma_{i}$ is a mapping from the set $\boldsymbol{R}^{2 N}$ of price vectors $(p, q)$ to the set $\{A, B, \emptyset\}$. Let $\sigma=\left(\sigma_{i}\right)_{i \in I}$ be the buyers' strategy profile, and for each choice profile $x=\left(x_{i}\right)_{i \in I}$ of buyers, let

$$
I_{A}(x)=\left\{i \in I: x_{i}=A\right\}, \quad \text { and } \quad I_{B}(x)=\left\{i \in I: x_{i}=B\right\}
$$

denote the set of buyers choosing seller $A$ and the set of buyers choosing $B$, respectively. If we denote by $\pi_{A}(p, q, \sigma)$ and $\pi_{B}(p, q, \sigma)$ the payoffs of sellers $A$ and $B$, respectively, under the strategy profile $(p, q, \sigma)$, then they are given by

$$
\begin{aligned}
& \pi_{A}(p, q, \sigma)=\sum_{i \in I_{A}(\sigma(p, q))} p_{i}, \\
& \pi_{B}(p, q, \sigma)=\sum_{i \in I_{B}(\sigma(p, q))} q_{i} .
\end{aligned}
$$

Given the price profile $(p, q)$, buyer $i$ 's payoff under the action profile $x$ depends on the number of his neighbors who consume the same good, i.e.,

$$
u_{i}(x)= \begin{cases}v^{\left|N_{i} \cap I_{A}(x)\right|}-p_{i} & \text { if } x_{i}=A, \\ v^{\left|N_{i} \cap I_{B}(x)\right|}-q_{i} & \text { if } x_{i}=B, \\ 0 & \text { if } x_{i}=\emptyset,\end{cases}
$$

and buyer $i$ 's payoff under the strategy profile $(p, q, \sigma)$ is written as:

$$
\pi_{i}(p, q, \sigma)=u_{i}(\sigma(p, q)) .
$$

A price vector $\left(p^{*}, q^{*}\right)$ and a strategy profile $\sigma=\left(\sigma_{i}\right)_{i \in I}$ together constitute a subgame perfect equilibrium (SPE) if given any price vector $(p, q) \in \boldsymbol{R}^{2 N}$, the action vector $\left(\sigma_{i}(p, q)\right)_{i \in I}$ is a Nash equilibrium of the subgame following $(p, q)$, and given $\sigma$, each component of the price vector $\left(p^{*}, q^{*}\right)$ is optimal against the other:

$$
\begin{aligned}
& \pi_{i}(p, q, \sigma(p, q)) \geq \pi_{i}\left(p, q, x_{i}, \sigma_{-i}(p, q)\right) \text { for every } x_{i}, i \text { and }(p, q), \\
& \pi_{A}\left(p^{*}, q^{*}, \sigma\left(p^{*}, q^{*}\right)\right) \geq \pi_{A}\left(p, q^{*}, \sigma\left(p, q^{*}\right)\right) \text { for every } p \\
& \pi_{B}\left(p^{*}, q^{*}, \sigma\left(p^{*}, q^{*}\right)\right) \geq \pi_{B}\left(p^{*}, q, \sigma\left(p^{*}, q\right)\right) \text { for every } q .
\end{aligned}
$$




\section{Nash Equilibrium in the Buyers' Game}

In this section, we fix the price vector $(p, q)$, and consider an equilibrium of the buyers' subgame following $(p, q)$. For the payoff function $u_{i}$ defined in (1), the simultaneous-move game $\left(I, S=\{A, B, \emptyset\}^{I},\left(u_{i}\right)_{i \in I}\right)$ among the buyers is a supermodular game when the set of actions of each buyer is endowed with the ordering $A \succ \emptyset \succ B$. It follows that the game has pure Nash equilibria that are maximal and minimal with respect to the partial ordering on $S$ induced by $\succ .{ }^{4}$ We refer to the maximal equilibrium as the $A$-maximal equilibrium and denote it by $x^{A}$, and the minimal equilibrium as the $B$-maximal equilibrium and denote it by $x^{B}$. By definition, for any NE $y$ and buyer $i, y_{i}=A$ implies $x_{i}^{A}=A$, and $y_{i}=B$ implies $x_{i}^{B}=B$.

We introduce some notation below in view of the fact that any NE must survive the iterative elimination of strictly dominated actions.

Define $T_{0}=\emptyset$ and recursively define the subsets of buyers $Y_{k}, Z_{k}, P_{k}, Q_{k}, R_{k}$, and $T_{k}$ as follows. For $k=0,1,2, \ldots$, define $Y_{k+1} \subset I \backslash T_{k}$ to be the maximal set such that

$$
\begin{aligned}
& Y_{k+1}=\left\{i \in I \backslash T_{k}:\right. \\
&\left.u_{i}\left(x_{T_{k}}^{*}, x_{Y_{k+1}}=(A, \ldots, A), x_{-T_{k}-Y_{k+1}}=(\emptyset, \ldots, \emptyset)\right) \geq 0\right\} .
\end{aligned}
$$

Given the action profile $x_{T_{k}}^{*}$ of buyers in set $T_{k}$, those buyers in $Y_{k+1}$ can collectively choose $A$ to enjoy non-negative payoffs from it. In other words, if $i \notin Y_{k+1}$, then $x_{i}=A$ is strictly dominated by $x_{i}=\emptyset$ for $i$. Note that maximality is well-defined since if $Y$ and $Y^{\prime}$ both satisfy (2), then $Y \cup Y^{\prime}$ also satisfies (2). If there is no such set, let $Y_{k+1}=\emptyset$. Likewise, define $Z_{k+1} \subset I \backslash T_{k}$ to be the maximal set of buyers who can collectively choose $B$ to enjoy non-negative payoffs from it:

$$
\begin{aligned}
Z_{k+1}=\{ & \left\{\in I \backslash T_{k}:\right. \\
& \left.u_{i}\left(x_{T_{k}}^{*}, x_{Z_{k+1}}=(B, \ldots, B), x_{-T_{k}-Z_{k+1}}=(\emptyset, \ldots, \emptyset)\right) \geq 0\right\} .
\end{aligned}
$$

If there is no such set, let $Z_{k+1}=\emptyset$. Again, if $i \notin Z_{k+1}$, then $x_{i}=B$ is strictly dominated by $x_{i}=\emptyset$ for $i$. Define

$$
R_{k+1}=\left(I \backslash T_{k}\right) \backslash\left(Y_{k+1} \cup Z_{k+1}\right)
$$

\footnotetext{
${ }^{4}$ See Topkis (1998)
} 
to be the set of buyers $i$ for whom $x_{i}=\emptyset$ is strictly dominant. Now define $P_{k+1} \subset$ $I \backslash T_{k}$ by

$$
\begin{aligned}
P_{k+1}=\left\{i \in I \backslash T_{k}:\right. & u_{i}\left(x_{T_{k}}^{*}, x_{i}=B, x_{-T_{k}-i}=(\emptyset, \ldots, \emptyset)\right) \\
& \left.>u_{i}\left(x_{T_{k}}^{*}, x_{Y_{k+1}}=(A, \ldots, A), x_{-T_{k}-Y_{k+1}}=(\emptyset, \ldots, \emptyset)\right)\right\},
\end{aligned}
$$

That is, if $i \in P_{n+1}$, buyer $i$ is strictly better off choosing $x_{i}=B$ than choosing $x_{i}=A$ or $\emptyset$ even if seller $A$ attracts all those buyers $j$ for whom $x_{j}=A$ is not strictly dominated by $x_{i}=\emptyset$. In other words, if $i \in P_{k+1}$, then $x_{i}=B$ is strictly dominant for $i$. Likewise, define $Q_{k+1} \subset I \backslash T_{k}$ to be the set of buyers $i$ for whom $x_{i}=A$ is strictly dominant:

$$
\begin{aligned}
Q_{k+1}=\left\{i \in I \backslash T_{k}:\right. & u_{i}\left(x_{T_{k}}^{*}, x_{i}=A, x_{-T_{k}-i}=(\emptyset, \ldots, \emptyset)\right) \\
& \left.>u_{i}\left(x_{T_{k}}^{*}, x_{Z_{k+1}}=(B, \ldots, B), x_{-T_{k}-Z_{k+1}}=(\emptyset, \ldots, \emptyset)\right)\right\} .
\end{aligned}
$$

1) If $P_{k+1}=Q_{k+1}=R_{k+1}=\emptyset$, then set $K=k$ and stop.

2) Otherwise, define

$$
x_{i}^{*}= \begin{cases}B & \text { if } i \in P_{k+1}, \\ A & \text { if } i \in Q_{k+1}, \\ \emptyset & \text { if } i \in R_{k+1} .\end{cases}
$$

and

$$
T_{k+1}=T_{k} \cup\left(P_{k+1} \cup Q_{k+1} \cup R_{k+1}\right) .
$$

If $T_{k+1}=I$, then set $K=k+1$ and stop. Otherwise, increase $k$ by one and start over.

Since the above process starts over only when at least one buyer has a strictly dominant action, the maximal number of rounds $K$ must satisfy $K \leq N$. For any NE $x$, we must have every buyer in $T_{K}$ choosing his iteratively strictly dominant action so that

$$
x_{T_{K}}=x_{T_{K}}^{*} .
$$

Therefore the possible difference between any pair of NE arises only for buyers in $I \backslash T_{K}$. The following proposition states that the $A$-maximal and $B$-maximal NE can be constructed by letting the maximal number of buyers choose $A$ or $B$ among those buyers. 
Proposition 1 Let $x^{A}$ and $x^{B}$ be defined by

$$
\begin{aligned}
& x^{A}=\left(x_{T_{K}}^{*}, x_{Y_{K+1}}=(A, \ldots, A), x_{-T_{K}-Y_{K+1}}=(\emptyset, \ldots, \emptyset)\right), \quad \text { and } \\
& x^{B}=\left(x_{T_{K}}^{*}, x_{Z_{K+1}}=(B, \ldots, B), x_{-T_{K}-Z_{K+1}}=(\emptyset, \ldots, \emptyset)\right) .
\end{aligned}
$$

Then $x^{A}$ and $x^{B}$ are the $A$-maximal and $B$-maximal $N E$, respectively.

Of course, when $T_{K}=I$ so that every buyer has a iteratively strictly dominant action, the NE is unique and given by $x^{A}=x^{B}$.

\section{Subgame Perfect Equilibrium}

We now turn to the original two-stage game including the sellers. The proposition below makes a simple observation that if a price vector $\left(p^{*}, q^{*}\right)$ is sustained in some SPE, then it must be sustained in an SPE in which the buyers choose an extreme response to either seller's deviation: If seller $A$ deviates from $p^{*}$, then all buyers coordinate on the $B$-maximal NE that least favors seller $A$, and vice versa. The proposition hence presents a bang-bang property of an SPE.

Proposition 2 For any network $G,\left(p^{*}, q^{*}\right)$ is an SPE price vector if and only if there exists buyers' strategy profile $\sigma$ such that $\left(p^{*}, q^{*}, \sigma\right)$ is an SPE and

$$
\sigma(p, q)= \begin{cases}\sigma^{B}(p, q) & \text { if } p \neq p^{*} \text { and } q=q^{*}, \\ \sigma^{A}(p, q) & \text { if } p=p^{*} \text { and } q \neq q^{*} .\end{cases}
$$

Consider next seller $A$ 's best response $p$ to $B$ 's price $q$ when the buyers play the $B$-maximal strategy $\sigma^{B}$. Since $\sigma^{B}(p, q)$ is a $B$-maximal NE for any $(p, q)$, seller $A$ can attract buyer $i$ if and only if $x_{i}=A$ is an iteratively strictly dominant action for buyer $i$ : $i \in \cup_{k=1}^{K} Q_{k}$, where $Q_{k}$ is as defined in (5). Hence,

$$
\pi_{A}\left(p, q . \sigma^{B}\right)=\sum_{k=1}^{K} \sum_{i \in Q_{k}} p_{i} .
$$

The following lemma shows that if seller $A$ 's price vector $p$ is a best response to $\left(q, \sigma^{B}\right)$, then no two buyers in $Q_{k}$ defined under $(p, q)$ are adjacent. In other words, in order to attract adjacent buyers $i$ and $j$, seller $A$ should approach them sequentially. Intuitively, this is because making choice $A$ dominant for both buyers simultaneously 
requires offering lower prices to both of them than making $x_{i}=A$ dominant for buyer $i$ first, then making $x_{j}=A$ dominant for buyer $j$ next conditional on the knowledge that $i$ chooses $x_{i}=A$.

Lemma 3 Let $\left(Q_{k}\right)_{k=1, \ldots, K}$ be as defined in (5) under the price vector $(p, q)$. If $p$ is a best response to $\left(q, \sigma^{B}\right)$, then for every $k=1, \ldots, K$,

$$
i, j \in Q_{k} \quad \Rightarrow \quad i \text { and } j \text { are not adjacent. }
$$

We now derive a key result that establishes a lower bound for each seller's equilibrium payoff given the price vector of the other seller. As mentioned in the Introduction, the argument is one of divide and conquer, where seller $A$, say, approaches each buyer sequentially according to some ordered list, and presents them with a price which makes the choice $A$ a dominant action given all his predecessors in the list choose $A$.

Formally, fix the price $q^{*}$ of seller $B$, and suppose that the buyers choose $A$ only when it is an iteratively strictly dominated action. Suppose further that seller $A$ makes an ordered list of all buyers $i_{1}, \ldots, i_{N}$. Seller $A$ first targets buyer $i_{1}$ by making it strictly dominant for him to choose $x_{i_{1}}=A$ by offering a sufficiently low price. In fact, seller $A$ needs to offer $p_{i_{1}}$ such that

$$
v^{0}-p_{i_{1}}>v^{d_{i_{1}}}-q_{i_{1}}^{*} \quad \text { and } \quad v^{0}-p_{i_{1}}>0
$$

or equivalently

$$
p_{i_{1}}<\min \left\{v^{0}-v^{d_{i_{1}}}+q_{i_{1}}^{*}, v^{0}\right\}
$$

to make $x_{i_{1}}=A$ strictly dominant. Let $H_{1}=\left\{i_{1}\right\}$. Seller $A$ next targets buyer $i_{2}$ by making $x_{i_{2}}=A$ strictly dominant. In this case, seller $A$ must offer $p_{i_{2}}$ such that

$$
p_{i_{2}}<\min \left\{v^{s_{i_{2}}}-v^{d_{i_{2}}-s_{i_{2}}}+q_{i_{2}}^{*}, v^{s_{i_{2}}}\right\},
$$

where $s_{i_{2}}=1$ if buyer $i_{2}$ is adjacent to $i_{1}$, and $=0$ otherwise. Let $H_{2}=\left\{i_{1}, i_{2}\right\}$. Proceeding iteratively, we see that against buyer $i_{k}$, seller $A$ must offer $p_{i_{k}}$ such that

$$
p_{i_{k}}<\min \left\{v^{s_{i_{k}}}-v^{d_{i_{k}}-s_{i_{k}}}+q_{i_{k}}^{*}, v^{s_{i_{k}}}\right\},
$$

where $s_{i_{k}}$ is the number of neighbors of $i_{k}$ in the set $H_{k-1}=\left\{i_{1}, \ldots, i_{k-1}\right\} . s_{i_{k}}$ can be thought of the externalities buyer $i_{k}$ can enjoy by choosing $A$ when those buyers 
in $H_{k-1}$ have already chosen $A$. On the other hand, $d_{i}-s_{i_{k}}$ is the externalities $i_{k}$ can enjoy from $B$ when those buyers in $I \backslash H_{k-1}$ still choose $B$. Note that for any list $i_{1}, \ldots, i_{N}$ of buyers,

$$
\sum_{k=1}^{N} s_{i_{k}}=M,
$$

where $M$ is the total number of links in $G$. Define $S$ by

$$
\begin{gathered}
S=\left\{s=\left(s_{i}\right)_{i \in I}: s_{i_{1}}=0 \text { and } s_{i_{k}}=\left|N_{i_{k}} \cap\left\{i_{1}, \ldots, i_{k-1}\right\}\right| \text { for } k \geq 2\right. \\
\text { for some relabeling } \left.\left(i_{1}, \ldots, i_{N}\right) \text { of buyers }\right\} .
\end{gathered}
$$

Note that if $s$ corresponds to the list $i_{1}, \ldots, i_{N}$, then $d-s=\left(d_{i}-s_{i}\right)_{i \in I}$ corresponds to the reversed list $i_{N}, \ldots, i_{1}$. Hence, if $s \in S$, then $d-s \in S$ as well. We also observe that

$$
H_{k} \subset \cup_{\ell=1}^{k} Q_{\ell}
$$

where $Q_{k}$ is as defined in (5) and equals the set of buyers $i$ for whom $x_{i}=A$ is iteratively strictly dominant in round $k$ of the iteration process under the price profile $\left(p, q^{*}\right)$. Hence, even if the buyers play the $B$-maximal equilibrium $\sigma^{B}$ that least favors seller $A, A$ can at least secure the payoff implied by the prices in (6). We hence have the following lemma that gives a lower bound for each seller's equilibrium payoff.

Lemma 4 If $\left(p^{*}, q^{*}, \sigma\right)$ is an $S P E$, then

$$
\begin{aligned}
& \pi_{A}\left(p^{*}, q^{*}, \sigma\right) \geq \max _{s \in S} \sum_{i=1}^{N} \min \left\{v^{s_{i}}-v^{d_{i}-s_{i}}+q_{i}^{*}, v^{s_{i}}\right\}, \\
& \pi_{B}\left(p^{*}, q^{*}, \sigma\right) \geq \max _{s \in S} \sum_{i=1}^{N} \min \left\{v^{s_{i}}-v^{d_{i}-s_{i}}+p_{i}^{*}, v^{s_{i}}\right\} .
\end{aligned}
$$

Figures 1 and 2 illustrate the discussion for the line network of three buyers. In Figure 1, seller $A$ approaches the buyers in the order $\left(i_{1}, i_{2}, i_{3}\right)=(1,3,2)$ when seller $B$ offers $q^{*}=\left(q_{1}^{*}, q_{2}^{*}, q_{3}^{*}\right)$ : When buyers 1 and 3 switch to $A$, their valuation of $A$ 's good is just $v^{0}$ (stand-alone value) since at that point they don't expect that buyer 2 will switch as well. On the other hand, when buyer 2 switches to $A$, he knows that both his neighbors will choose $A$, and he expects that $A$ 's good is worth $v^{2}$. Hence, 


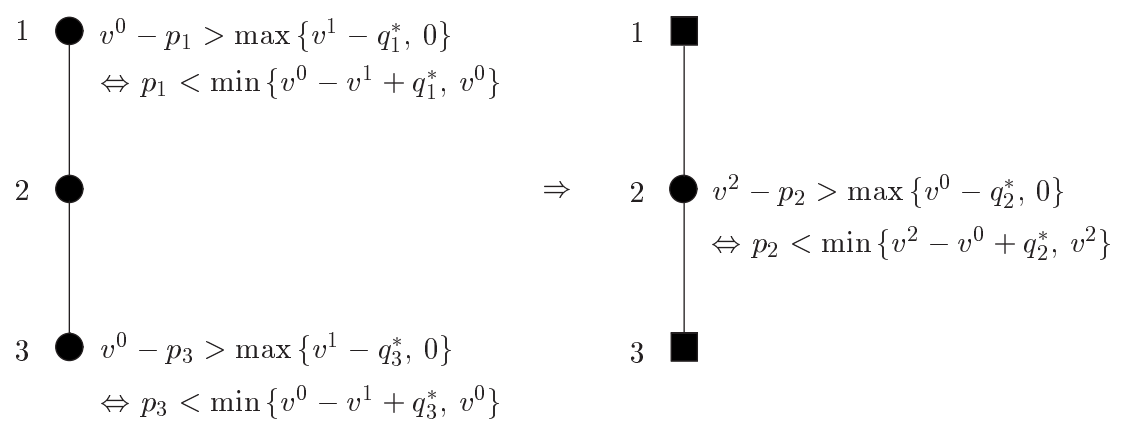

Figure 1: Divide-and-conquer by seller $A$ with $\left(i_{1}, i_{2}, i_{3}\right)=(1,3,2)$. $\Leftrightarrow p_{2}<\min \left\{v^{0}-v^{2}+q_{2}^{*}, v^{0}\right\}$

3
$1 \bigcirc v^{1}-p_{1}>\max \left\{v^{0}-q_{1}^{*}, 0\right\}$ $\Leftrightarrow p_{1}<\min \left\{v^{1}-v^{0}+q_{1}^{*}, v^{1}\right\}$

$v^{1}-p_{3}>\max \left\{v^{0}-q_{3}^{*}, 0\right\}$

$\Leftrightarrow p_{3}<\min \left\{v^{1}-v^{0}+q_{3}^{*}, v^{1}\right\}$

Figure 2: Divide-and-conquer by seller $A$ with $\left(i_{1}, i_{2}, i_{3}\right)=(2,1,3)$.

even if the buyers play the $B$-maximal equilibrium $\sigma^{B}$, seller $A$ 's divide-and-conquer strategy with $\left(i_{1}, i_{2}, i_{3}\right)=(1,3,2)$ is profitable if

$$
\begin{aligned}
\min \left\{v^{0}-v^{1}+q_{1}^{*}, v^{0}\right\} & +\min \left\{v^{0}-v^{1}+q_{3}^{*}, v^{0}\right\} \\
& +\min \left\{v^{2}-v^{0}+q_{2}^{*}, v^{2}\right\}>0 .
\end{aligned}
$$

Likewise, his divide-and-conquer strategy with $\left(i_{1}, i_{2}, i_{3}\right)=(2,1,3)$ illustrated in Figure 2 is profitable if

$$
\begin{aligned}
\min \left\{v^{0}-v^{2}+q_{2}^{*}, v^{0}\right\} & +\min \left\{v^{1}-v^{0}+q_{1}^{*}, v^{1}\right\} \\
& +\min \left\{v^{1}-v^{0}+q_{3}^{*}, v^{1}\right\}>0,
\end{aligned}
$$

and that with $\left(i_{1}, i_{2}, i_{3}\right)=(1,2,3)$ is profitable if

$$
\begin{aligned}
\min \left\{v^{0}-v^{1}+q_{1}^{*}, v^{0}\right\} & +\min \left\{v^{1}-v^{1}+q_{2}^{*}, v^{1}\right\} \\
& +\min \left\{v^{1}-v^{0}+q_{3}^{*}, v^{1}\right\}>0 .
\end{aligned}
$$




\section{Marginal Cost Pricing}

When there are no consumption externalities $0<v^{0}=\cdots=v^{N-1}$, it is clear that a subgame perfect equilibrium price $\left(p^{*}, q^{*}\right)$ is unique and equal to the marginal cost: $\left(p^{*}, q^{*}\right)=(0,0)$. In this section, we will examine if and how this result can be extended when there are externalities.

Let $D=D(G)$ be the highest degree in $G$ :

$$
D(G)=\max _{i \in I} d_{i}(G)
$$

For the network $G$, hence, the relevant levels of externalities are $\left(v^{0}, \ldots, v^{D}\right)$. We say that the externalities $\left(v^{0}, \ldots, v^{D}\right)$ are linear if there exists $h>0$ such that

$$
v^{k}=k h \quad \text { for every } k=0,1, \ldots, D \text {. }
$$

Note in particular that the stand-alone value $v^{0}$ is zero when the externalities are linear. In this sense, linearity implies pure network externalities and violates the formulation of weak externalities in Jullien $(2011) .^{5}$

Proposition 5 Let $G$ be an arbitrary buyer network. Under the linear externalities $\left(v^{0}, \ldots, v^{D}\right),\left(p^{*}, q^{*}\right)=(0,0)$ is an SPE price vector.

We next consider some generic property of externalities. As will be seen, whether or not the marginal cost can be an equilibrium price depends crucially on the configuration of the buyer network in this case. Specifically, for $S$ defined in (7), suppose that the externalities $\left(v^{0}, \ldots, v^{D}\right)$ satisfy the following condition:

$$
s \in S \text { and } d-s \text { is not a permutation of } s \Rightarrow \sum_{i=1}^{N} v^{s_{i}} \neq \sum_{i=1}^{N} v^{d_{i}-s_{i}} .
$$

(11) implies that the sum of externalities over buyers are different between the two goods when seller $A$ attracts buyers by offering prices as described in (6). The set of $\left(v^{0}, \ldots, v^{D}\right)$ satisfying $(11)$ is generic in the set

$$
\left\{\left(v^{0}, \ldots, v^{D}\right): 0<v^{0} \leq \cdots \leq v^{D}\right\}
$$

of all externalities.

\footnotetext{
${ }^{5}$ Assumption 1 of Jullien (2011).
} 
Lemma 4 implies that a seller's equilibrium payoff is closely linked to the value of

$$
\max _{s \in S} \sum_{i=1}^{N}\left(v^{s_{i}}-v^{d_{i}-s_{i}}\right) .
$$

It turns out that whether this quantity is positive or not under (11) depends crucially on the network configuration as seen in the following lemma.

Lemma 6 Suppose that the externalities $v=\left(v^{0}, \ldots, v^{D}\right)$ satisfy (11). If the buyer network $G$ is neither cyclic nor complete, then

$$
\max _{s \in S} \sum_{i=1}^{N}\left(v^{s_{i}}-v^{d_{i}-s_{i}}\right)>0 .
$$

The following lemma, which readily follows from Lemmas 4 and 6 , provides some key observations on equilibrium pricing.

Lemma 7 Suppose that $\left(p^{*}, q^{*}, \sigma\right)$ is an SPE for the buyer network $G$ which is neither complete nor cyclic, and that the externalities $v=\left(v^{0}, \ldots, v^{D}\right)$ satisfy (11). Then

a) $\pi_{A}\left(p^{*}, q^{*}, \sigma\right)=0 \Rightarrow \min _{i} q_{i}^{*}<0$.

b) $\pi_{A}\left(p^{*}, q^{*}, \sigma\right) \leq \sum_{i} q_{i}^{*} \Rightarrow \max _{i} q_{i}^{*}>v^{0}$.

c) $I_{B}\left(\sigma\left(p^{*}, q^{*}\right)\right)=I \Rightarrow \max _{i} q_{i}^{*}>v^{0}, \min _{i}\left(v^{d_{i}}-q_{i}^{*}\right) \geq v^{0}$, and $v^{D}>2 v^{0}$.

While the first two statements of Lemma 7 are true regardless of whether the market is monopolized or segmented in equilibrium, the implications of the lemma are seen most clearly for a monopolization equilibrium. Suppose that $G$ is neither cyclic nor complete, and that seller $B$ captures the entire market in equilibrium: $I_{B}\left(\sigma\left(p^{*}, q^{*}\right)\right)=I$. Then seller $B$ must subsidize at least one buyer, and must charge some buyer strictly above the stand-alone value:

$$
\min _{i} q_{i}^{*}<0 \leq v^{0}<\max _{i} q_{i}^{*}<v^{D}-v^{0} .
$$

Furthermore, for any such equilibrium to exist, the externalities cannot be too small: $v^{D}>2 v^{0}$. This is a non-trivial restriction for networks in which every buyer has a small degree as in line networks. We summarize this observation as a proposition below. 
Proposition 8 Let a buyer network $G$ be given and the externalities $v=\left(v^{0}, \ldots, v^{D}\right)$ satisfy (11). Then there exists no SPE in which one of the sellers monopolizes the market by charging the same price to every buyer.

The impossibility of uniform pricing is counter-intuitive in networks which are not cyclic or complete, but are symmetric with respect to every buyer. For example, buyer locations are exactly symmetric in the 4-regular network depicted in Figure 3.

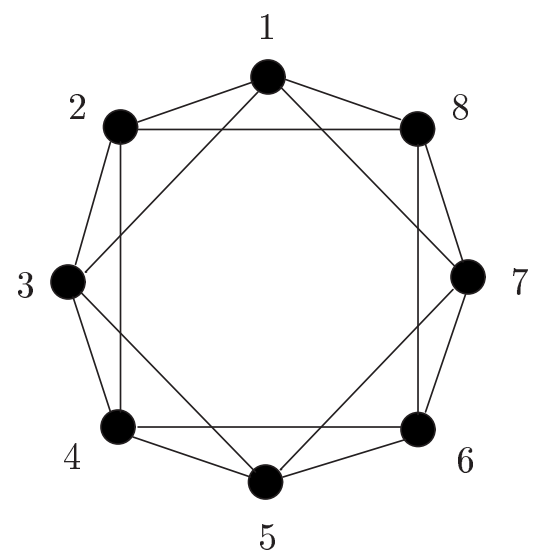

Figure 3: 4-regular network with eight buyers

We are now ready to state our main result on marginal cost pricing. Suppose that both sellers offer zero to all the buyers. In this case, both sellers' payoffs equal zero regardless of whether or not they capture a positive portion of the market. Hence, this price profile cannot be an equilibrium by Lemma 7(a) unless the network is complete or cyclic. The following proposition shows that when the network is complete or cyclic, there indeed exists an SPE of the type presented in Proposition 2 in which both sellers offer zero.

Proposition 9 Let a buyer network $G$ be given and the externalities $v=\left(v^{0}, \ldots, v^{D}\right)$ satisfy $(11) .\left(p^{*}, q^{*}\right)=(0,0)$ is an SPE price vector if and only if $G$ is either cyclic or complete.

For illustration of the impossibility of marginal cost pricing, return to the example of the three-buyer line network depicted in Figures 1 and 2. Suppose that 
$q^{*}=0$. In this case, we have

$$
\begin{gathered}
(9) \Leftrightarrow 2 v^{1}-v^{2}-v^{0}<0, \\
(10) \Leftrightarrow 2 v^{1}-v^{2}-v^{0}>0 .
\end{gathered}
$$

Hence, if

$$
2 v^{1} \neq v^{2}+v^{0}
$$

seller $A$ can profitably divide and conquer the buyers against $q^{*}=0$. Note that (13) corresponds to (12) in Lemma 6: It fails under the linear externalities $v^{0}=0$, $v^{1}=h$ and $v^{2}=2 h$, but is true under generic specifications of $v^{0}, v^{1}$ and $v^{2}$.

\section{Monopolization on a Bipartite Network}

The results in the preceding section suggest that some form of discriminatory pricing is inevitable in equilibrium. A natural question then is on the form of equilibrium price discrimination. Interesting related questions are (1) which buyers are the "weak link" in the network that need to be protected, and (2) which buyers can be squeezed for more profits. Since it appears difficult to provide general answers to these questions, we will restrict attention to certain classes of networks for the identification of an equilibrium. In this section, we identify a class of networks in which monopolization takes place in equilibrium.

Our analysis in what follows assumes that the externalities are approximately linear in the following sense: For $h>0$, the externalities $\left(v^{0}, \ldots, v^{D}\right)$ are $\varepsilon$-close to linear if

$$
\left|v^{k}-k h\right|<\varepsilon \text { for } k=0,1, \ldots, D .
$$

Since the condition holds for any $\varepsilon>0$ when the externalities are exactly linear, our conclusions under approximate linearity hold with no change in models of linear externalities. In conjunction with Proposition 5, then, this implies the multiplicity of equilibria in these markets.

The buyer network is bipartite if the buyer set $I$ is partitioned into two disjoint subsets $I_{1}$ and $I_{2}$ such that every neighbor of $i \in I_{1}$ belongs to $I_{2}$ and every neighbor of $i \in I_{2}$ belongs to $I_{1}$. Line and star networks are simple examples of a bipartite network. For example, the line network in Figures 1 and 2 is bipartite with the partition $I_{1}=\{1,3\}$ and $I_{2}=\{2\}$. A cycle network with an even number of buyers is also bipartite. A bipartite network is complete if every buyer in $I_{1}$ is linked to every buyer in $I_{2}$. 
Bipartite networks are particularly important in their connection to two-sided markets. For example, we can think of $I_{1}$ as the set of sellers and $I_{2}$ as the set of buyers of a certain good. In this case, the sellers $A$ and $B$ are interpreted as the platforms that offer marketplace to these sellers and buyers, and their prices are interpreted as participation fees into their platforms. A complete bipartite network corresponds to a two-sided market in which each agent finds more value in a given platform whenever more agents on the other side participate in the same platform. Our conclusion on a bipartite network translates to that on a two-sided market where two platforms compete.

Proposition 10 Suppose that the buyer network $G$ is bipartite. For any $h>0$, there exists $\bar{\varepsilon}>0$ such that if the externalities are $\varepsilon$-close to h-linear for $\varepsilon<\bar{\varepsilon}$, then there exists an $\operatorname{SPE}\left(p^{*}, q^{*}, \sigma\right)$ in which one seller captures all the buyers.

The equilibrium constructed in the proof is described as follows: Let $I_{1}$ and $I_{2}$ be the partition of the buyer set, and suppose that seller $B$ captures the market. Seller $B$ offers $q_{i}=v^{d_{i}}-v^{0}$ to each buyer $i$ in set $I_{1}$ and $q_{i}=v^{0}-v^{d_{i}}$ to each buyer $i$ in set $I_{2}$ provided that these prices lead to a non-negative payoff. ${ }^{6}$ In other words, the monopolizing seller taxes every buyer on one side, and subsidizes every buyer on the other side. Seller $A$ offers the same price to each buyer as seller $B$. When either seller deviates, the buyers play the extreme equilibrium which is least favorable to the deviating seller as in Proposition 2. It is shown that this price vector leaves no room for seller $A$ to profitably attract any buyers. Figure 4 illustrates the equilibrium pricing of Proposition 10 in a star network with five buyers when the externalities satisfy approximate linearity and

$$
v^{4}-v^{0} \geq 4\left(v^{1}-v^{0}\right) .
$$

It can be seen that the hub buyer 1 is charged a positive price whereas all the peripheral buyers are subsidized. In other words, the subsidies to the peripheral buyers are a protection against the inducement by the other seller. Since (14) holds when the externalities are marginally increasing, we can understand this pricing behavior from the fact that it is relatively more difficult for the other seller to entice the hub buyer. When the inequality (14) is reversed, then the prices are (-1) times those listed in Figure 4. In this case of marginally decreasing externalities, hence,

\footnotetext{
${ }^{6}$ Recall that $d_{i}$ denotes the degree of buyer $i$. If these prices lead to a negative payoff, the equilibrium prices are simply $-q_{i}$ for each $i$.
} 
the hub buyer needs to be protected as it is relatively easier for the other seller to entice him. As seen in this example, the specification of externalities determines which buyer(s) should be protected with subsidies.

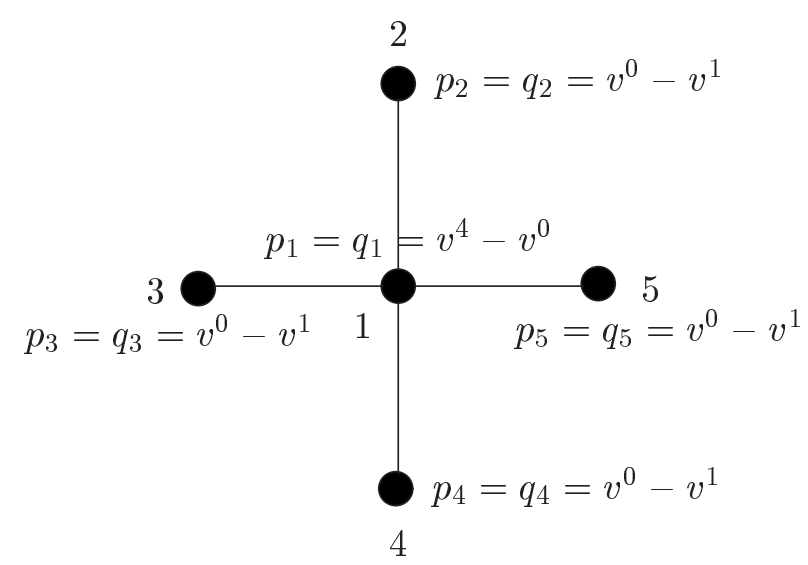

Figure 4: Monopolization through discriminatory pricing on a star network when $v^{4}-v^{0} \geq 4\left(v^{1}-v^{0}\right)$.

Decreasing or increasing marginal externalities also have the following implications for the pricing in a complete bipartite network: Under increasing marginal externalities, any buyer in a complete bipartite network is subsidized in equilibrium if and only if his subset of buyers is larger than the other subset. The opposite holds under decreasing marginal externalities.

Corollary 11 Suppose that the network is complete bipartite with partition $\left(I_{1}, I_{2}\right)$ such that $\left|I_{1}\right| \leq\left|I_{2}\right|$. For any $h>0$, there exists $\bar{\varepsilon}>0$ such that the following hold for $\varepsilon<\bar{\varepsilon}$ :

a) (increasing marginal externalities) If

$$
h-\varepsilon \leq v^{1}-v^{0} \leq v^{2}-v^{1} \leq \cdots \leq v^{D}-v^{D-1} \leq h+\varepsilon,
$$

then there exists an $\operatorname{SPE}(p, q, \sigma)$ such that $p_{i}=q_{i}>0$ for every $i \in I_{1}$ and $p_{i}=q_{i}<0$ for every $i \in I_{2}$.

b) (decreasing marginal externalities) If

$$
h-\varepsilon \leq v^{D}-v^{D-1} \leq \cdots \leq v^{2}-v^{1} \leq v^{1}-v^{0} \leq h+\varepsilon,
$$

then there exists an $\operatorname{SPE}(p, q, \sigma)$ such that $p_{i}=q_{i}<0$ for every $i \in I_{1}$ and $p_{i}=q_{i}>0$ for every $i \in I_{2}$. 


\section{Segmentation on a Separable Network}

Under the same assumption of approximately linear externalities as in the previous section, we now examine the possibility of an equilibrium in which market segmentation takes place. For this, we consider a class of buyer networks that have roughly the opposite property as the bipartite networks introduced in the previous section: In this class of networks, the buyer set is again partitioned into two disjoint subsets, but each buyer has at least as many neighbors in the same subset than in the other subset. Formally, the buyer network is separable if there exists a two-way partition $\left(I_{1}, I_{2}\right)$ of the set $I$ of buyers such that for $m, n=1,2$, and $m \neq n$,

$$
\begin{aligned}
& \left|N_{i} \cap I_{n}\right| \geq\left|N_{i} \cap I_{m}\right| \text { for every } i \in I_{n}, \text { and } \\
& \left|N_{i} \cap I_{n}\right|>\left|N_{i} \cap I_{m}\right| \text { for some } i \in I_{n} .
\end{aligned}
$$

Intuitively, in a separable network with partition $\left(I_{1}, I_{2}\right)$, we can classify buyers in $I_{1}$ or $I_{2}$ into core and peripheral buyers: The core buyers are those who have strictly more neighbors in the same set than in the other set, while the peripheral buyers have as many neighbors in the same set as in the other set. We can see that any line network with four or more buyers is separable: For example, a line network of four or more buyers is separable. The regular network in Figure 3 is also separable when we take $I_{1}=\{1,2,3,4\}$ and $I_{2}=\{5,6,7,8\}$. Buyer 2 and 3 are core buyers for $I_{1}$ and buyers 6 and 7 are core buyers for $I_{2}$.

Proposition 12 Suppose that $G$ is separable. For any $h>0$, there exists $\bar{\varepsilon}>0$ such that if the externalities are $\varepsilon$-close to h-linear for $\varepsilon<\bar{\varepsilon}$, there exists an SPE in which buyers in $I_{1}$ choose seller $A$ and buyers in $I_{2}$ choose seller $B$.

The proof of this proposition constructs an equilibrium in which each seller charges a small but positive price to one of the core buyers in his segment of the market. Specifically, recalling that $s$ is a sequence of degrees of externalities as defined in (7), we specify the price to be charged to this core buyer by

$$
\delta=\max _{s \in S} \sum_{i=1}^{N}\left(v^{s_{i}}-v^{d_{i}-s_{i}}\right),
$$

which is strictly positive for generic externalities (Lemma 6), but is small for approximately linear externalities. Each seller charges zero to all other buyers in their segment of the market. Figure 5 illustrates the equilibrium for a line network of four 


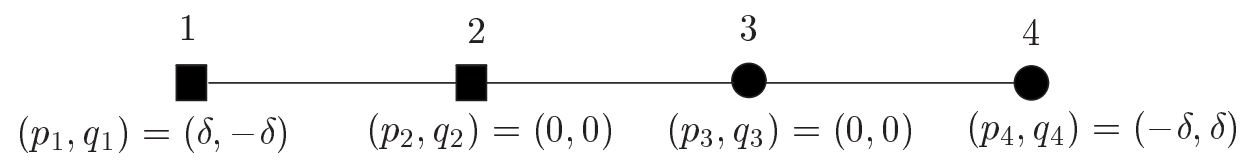

Figure 5: Segmentation on a line network $\left(\delta=\left|v^{2}+v^{1}-2 v^{0}\right|>0\right)$ : A captures $\{1,2\}$ and $B$ captures $\{3,4\}$.

buyers. ${ }^{7}$ As in Proposition 2, any deviation by either seller results in the extreme equilibrium that is least favorable to the deviating seller. Each core buyer who is charged the positive price will not switch to the other seller since he enjoys strictly higher externalities under the present seller. Furthermore, each seller enjoys strictly positive profits in equilibrium, and has no incentive to engage in divide-and-conquer taking advantage of the non-generic externalities as in the case of marginal cost pricing.

\section{Conclusion}

In this paper, we formulate a model of price competition between two sellers when each one of their goods exhibits local network externalities as represented by a graphtheoretic network of buyers. We show that whether a given price profile is consistent with a subgame perfect equilibrium of the two-stage game depends crucially on the exact specifications of network structure and externalities. In the non-generic case of linear externalities, the marginal cost pricing of both sellers quoting zero to every buyer is consistent with an SPE for any network. In the generic specification of externalities, however, it is consistent with an SPE if and only if the network is either cyclic or complete. That is, in any other networks, some form of price discrimination is expected even if every buyer has exactly symmetric locations in those networks. Given these results, we proceed to the identification of an SPE when the externalities are approximately linear. In a bipartite network which corresponds to a two-sided market, we show that there exists an SPE in which one of the sellers monopolizes the market by charging a positive price to every buyer on one side, and a negative price to every buyer on the other side. The pricing strategy there gives us a hint as to which buyer needs to be protected from the inducement by the other

\footnotetext{
${ }^{7}$ As seen in Figure 5, each seller charges $-\delta$ to the core buyer in the other segment who is charged $\delta$ by the other seller. This is to make the sum of the prices of each seller equal to zero.
} 
seller. In a separable network in which each buyer has more neighbors on his side than on the other side, on the other hand, we show that there exists an equilibrium in which the two sellers segment the market.

In the present model, the goods of the two sellers are assumed symmetric and incompatible with each other. A natural extension would involve introducing asymmetry or a positive degree of compatibility between them. It would also be interesting to study endogenous determination of compatibility levels by the sellers. Although some of these issues are investigated in the literature, ${ }^{8}$ it will be useful to examine them under the alternative specifications of externalities and equilibrium as in the present paper.

\section{Appendix I: Proofs}

Proof of Proposition 1. We show that $x^{A}$ is an $A$-maximal NE. The symmetric argument shows that $x^{B}$ is a $B$-maximal NE. We begin with the following lemma.

Lemma 13 a) $u_{i}\left(x^{A}\right) \geq 0$ for every $i$.

b) For any $n,\left\{i \in I \backslash T_{n}: x_{i}^{A}=A\right\} \subset Y_{n+1}$ and $\left\{i \in I \backslash T_{n}: x_{i}^{A}=B\right\} \subset Z_{n+1}$.

c) For any $n, J \subset I \backslash T_{n}$, and $y_{J}$ such that $u_{i}\left(y_{J}, x_{-J}^{A}\right) \geq 0$ for every $i \in J$,

$$
\left\{i \in J: y_{i}=A\right\} \subset Y_{n+1} \quad \text { and } \quad\left\{i \in J: y_{i}=B\right\} \subset Z_{n+1} \text {. }
$$

Proof of Lemma 13. a) Suppose that $i \in P_{n+1}$ for some $n$. Then

$$
\begin{aligned}
u_{i}\left(x^{A}\right) & =u_{i}\left(x_{T_{n}}^{A}, x_{i}=B, x_{-T_{n}-i}^{A}\right) \\
& \geq u_{i}\left(x_{T_{n}}^{A}, x_{i}=B, x_{-T_{n}-i}=(\emptyset, \ldots, \emptyset)\right) \\
& >u_{i}\left(x_{T_{n}}^{A}, x_{Y_{n+1}}=(A, \ldots, A), X_{-T_{n}-Y_{n+1}}=(\emptyset, \ldots, \emptyset)\right) \\
& \geq 0 .
\end{aligned}
$$

The proof is similar if $i \in Q_{n+1}$. If $i \in R_{n+1}$ or $i \in I \backslash T_{n}$ for $n$ such that $P_{n+1}=$ $Q_{n+1}=R_{n+1}=\emptyset$, then the inequality follows from the definition of $x^{A}$.

\footnotetext{
${ }^{8}$ See Jullien (2011).
} 
b) Denote $K=\left\{i \in I \backslash T_{n}: x_{i}^{A}=A\right\}$. Then for any $i \in K$, we have

$$
\begin{aligned}
& u_{i}\left(x_{T_{n}}^{A}, x_{K \cup Y_{n+1}}=(A, \ldots, A), x_{-T_{n}-K-Y_{n+1}}=(\emptyset, \ldots, \emptyset)\right) \\
& \geq u_{i}\left(x_{T_{n}}^{A}, x_{K}=(A, \ldots, A), x_{-T_{n}-K}=(\emptyset, \ldots, \emptyset)\right) \\
& =u_{i}\left(x^{A}\right) \geq 0,
\end{aligned}
$$

and for any $i \in Y_{n+1}$,

$$
\begin{aligned}
& u_{i}\left(x_{T_{n}}^{A}, x_{K \cup Y_{n+1}}=(A, \ldots, A), x_{-T_{n}-K-Y_{n+1}}=(\emptyset, \ldots, \emptyset)\right) \\
& \geq u_{i}\left(x_{T_{n}}^{A}, x_{Y_{n+1}}=(A, \ldots, A), x_{-T_{n}-Y_{n+1}}=(\emptyset, \ldots, \emptyset)\right) \\
& \geq 0 .
\end{aligned}
$$

This contradicts the maximality of $Y_{n+1}$.

c) Denote $K=\left\{i \in J: y_{i}=A\right\}$. Suppose that $K \not \subset Y_{n+1}$. Then for $i \in K$,

$$
\begin{aligned}
& u_{i}\left(x_{T_{n}}^{A}, x_{K \cup Y_{n+1}}=(A, \ldots, A), x_{-T_{n}-Y_{n+1}-K}=(\emptyset, \ldots, \emptyset)\right) \\
& \geq u_{i}\left(x_{T_{n}}^{A}, x_{J}, x_{-T_{n}-J}^{A}\right) \\
& =u_{i}\left(x_{-J}^{A}, x_{J}\right) \\
& \geq 0
\end{aligned}
$$

where the first inequality follows from Lemma 13(b), and for any $i \in Y_{n+1}$,

$$
\begin{aligned}
& u_{i}\left(x_{T_{n}}^{A}, x_{K \cup Y_{n+1}}=(A, \ldots, A), x_{-T_{n}-Y_{n+1}-K}=(\emptyset, \ldots, \emptyset)\right) \\
& u_{i}\left(x_{T_{n}}^{A}, x_{Y_{n+1}}=(A, \ldots, A), x_{-T_{n}-Y_{n+1}}=(\emptyset, \ldots, \emptyset)\right) \\
& \geq 0 .
\end{aligned}
$$

This again contradicts the maximality of $Y_{n+1}$.

We now return to the proof of Proposition 1.

1) $x^{A}$ is a NE.

Since $u_{i}\left(x^{A}\right) \geq 0$ by Lemma $13(\mathrm{a}), x_{i}^{\prime}=\emptyset$ cannot be a profitable deviation for any $i$, and moreover a profitable deviation, if any, must yield a strictly positive payoff.

Take any $i \in P_{n+1}$ so that $x_{i}^{A}=B$, and consider a deviation $x_{i}^{\prime}=A$. If $u_{i}\left(x_{i}^{\prime}, x_{-i}^{A}\right) \geq 0$, then $i \in Y_{n+1}$ by Lemma 13(c) and hence

$$
\begin{aligned}
u_{i}\left(x_{i}^{\prime}, x_{-i}^{A}\right) & \leq u_{i}\left(x_{T_{n}}^{A}, x_{Y_{n+1}}=(A, \ldots, A), x_{-Y_{n+1}}=(\emptyset, \ldots, \emptyset)\right) \\
& <u_{i}\left(x_{T_{n}}^{A}, x_{i}^{A}, x_{-T_{n}-i}=(\emptyset, \ldots, \emptyset)\right) \\
& \leq u_{i}\left(x^{A}\right) .
\end{aligned}
$$


Hence the deviation is not profitable. Likewise, no profitable deviation exists for $i \in Q_{n+1}$. Suppose next that $i \in R_{n+1}$ so that $x_{i}^{A}=\emptyset . x_{i}^{\prime}=A$ is not profitable since $i \in R_{n+1}$ implies that $i \notin Y_{n+1}$ and hence $u_{i}\left(x_{i}^{\prime}, x_{-i}^{A}\right)<0$ by Lemma 13(c). Likewise, the deviation $x_{i}^{\prime}=B$ is not profitable. Finally, suppose that $i \in I \backslash T_{n}$ and that $P_{n+1}=Q_{n+1}=R_{n+1}=\emptyset$. In this case, $x_{i}^{A}=A$ if $i \in Y_{n+1}$ and $x_{i}^{A}=\emptyset$ otherwise. If $x_{i}^{\prime}=B$, then

$$
\begin{aligned}
u_{i}\left(x_{i}^{\prime}, x_{-i}^{A}\right) & =u_{i}\left(x_{T_{n}}^{A}, x_{i}^{\prime}, x_{-T_{n}-i}^{A}\right) \\
& =u_{i}\left(x_{T_{n}}^{A}, x_{i}^{\prime}, x_{-T_{n}-i}=(\emptyset, \ldots, \emptyset)\right) \\
& \leq u_{i}\left(x_{T_{n}}^{A}, x_{Y_{n+1}}=(A, \ldots, A), x_{-T_{n}-Y_{n+1}}=(\emptyset, \ldots, \emptyset)\right) \\
& =u_{i}\left(x^{A}\right)
\end{aligned}
$$

where the inequality follows since $i \notin P_{n+1}$. Hence, $x_{i}^{\prime}=B$ is not a profitable deviation. If $i \in I \backslash T_{n} \backslash Y_{n+1}$ and $x_{i}^{\prime}=A$, then $u_{i}\left(x_{i}^{\prime}, x_{-i}^{A}\right)<0$ by Lemma 13(c).

2) $x^{A}$ is $A$-maximal.

Take any NE $y$. Clearly, $u_{i}(y) \geq 0$ for every $i$. We first show that $y_{i}=x_{i}^{A}$ if $i \in T_{1}$. To see that $y_{i}=B$ for any $i \in P_{1}$, suppose $y_{i}=A$. Then by setting $n=0$ and $J=I$ in Lemma $13\left(\right.$ c), we see that $\left\{i: y_{i}=A\right\} \subset Y_{1}$ so that

$$
\begin{aligned}
u_{i}\left(y_{i}, y_{-i}\right) & \leq u_{i}\left(x_{Y_{1}}=(A, \ldots, A), x_{-Y_{1}}=(\emptyset, \ldots, \emptyset)\right) \\
& <u_{i}\left(x_{i}=B, x_{-i}=(\emptyset, \ldots, \emptyset)\right) \\
& \leq u_{i}\left(x_{i}=B, y_{-i}\right),
\end{aligned}
$$

where the second inequality follows from the definition of $P_{1}$. Hence $x_{i}=B$ is a profitable deviation. Likewise, $y_{i}=A$ holds for any $i \in Q_{1}$. If $i \in R_{1}$, then $y_{i}=\emptyset$ must hold since $i \notin Y_{1} \cup Z_{1}$.

As an induction hypothesis, suppose that $y_{i}=x_{i}^{A}$ if $i \in T_{n}$. We show that $y_{i}=x_{i}^{A}$ if $i \in T_{n+1} \backslash T_{n}$. If $i \in P_{n+1}$, then $y_{i}=B$ : If $y_{i}=A$, then $\left\{i \in I \backslash T_{n}: y_{i}=\right.$ $A\} \subset Y_{n+1}$ by Lemma 13(c) (set $J=I \backslash T_{n}$ ) so that

$$
\begin{aligned}
u_{i}\left(y_{i}, y_{-i}\right) & \leq u_{i}\left(x_{T_{n}}^{A}, x_{Y_{n+1}}=(A, \ldots, A), x_{-T_{n}-Y_{n+1}}=(\emptyset, \ldots, \emptyset)\right) \\
& <u_{i}\left(x_{T_{n}}^{A}, x_{i}=B, x_{-T_{n}-i}=(\emptyset, \ldots, \emptyset)\right) \\
& \leq u_{i}\left(x_{i}=B, y_{-i}\right)
\end{aligned}
$$

where the second inequality follows from the definition of $P_{n+1}$. Hence $x_{i}=B$ is a profitable deviation. Likewise, $y_{i}=A$ for any $i \in Q_{n+1}$. If $i \in R_{n+1}$, then $y_{i}=\emptyset$ 
must hold since $i \notin Y_{n+1} \cup Z_{n+1}$. Finally, if $i \in I \backslash T_{n}$ and $P_{n+1}=Q_{n+1}=R_{n+1}=\emptyset$, then $y_{i}=A$ implies $i \in Y_{n+1}$ by Lemma 13(c), but $x_{i}^{A}=A$ for any such $i$ by definition. We can therefore conclude that $x^{A}$ is an $A$-maximal NE.

Proof of Proposition 2. If there exists such a strategy profile $\sigma$ of buyers, then $\left(p^{*}, q^{*}\right)$ is clearly an SPE price vector. Conversely, suppose that $\left(p^{*}, q^{*}\right)$ is an SPE price vector. Then there exists $\hat{\sigma}$ such that $\left(p^{*}, q^{*}, \hat{\sigma}\right)$ is an SPE. Define $\sigma$ as follows:

$$
\sigma(p, q)= \begin{cases}\hat{\sigma}(p, q) & \text { if }(p, q)=\left(p^{*}, q^{*}\right), \text { or } p \neq p^{*} \text { and } q \neq q^{*}, \\ \sigma^{A}(p, q) & \text { if } p=p^{*} \text { and } q \neq q^{*}, \\ \sigma^{B}(p, q) & \text { if } p \neq p^{*} \text { and } q=q^{*} .\end{cases}
$$

Then $\left(p^{*}, q^{*}, \sigma\right)$ is an SPE: The definition of $\sigma^{B}$ and the equilibrium property of $\hat{\sigma}$ together imply

$$
\pi_{A}\left(p, q^{*}, \sigma\right) \leq \pi_{A}\left(p, q^{*}, \hat{\sigma}\right) \leq \pi_{A}\left(p^{*}, q^{*}, \hat{\sigma}\right)=\pi_{A}\left(p^{*}, q^{*}, \sigma\right) .
$$

Likewise, the definition of $\sigma^{A}$ and the equilibrium property of $\hat{\sigma}$ together imply $\pi_{B}\left(p^{*}, q, \sigma\right) \leq \pi_{B}\left(p^{*}, q^{*}, \sigma\right)$.

Proof of Lemma 3. For simplicity, let $k=K$ and suppose to the contrary that $1,2 \in Q_{K}$ and 1 and 2 are adjacent. Then it must be the case that

$$
v^{\alpha_{1}^{K}}-p_{1}>\max \left\{v^{d_{1}-\alpha_{1}^{K}}-q_{1}, 0\right\} \quad \text { and } \quad v^{\alpha_{2}^{K}}-p_{2}>\max \left\{v^{d_{2}-\alpha_{2}^{K}}-q_{2}, 0\right\},
$$

where

$$
\alpha_{1}^{K}=\left|N_{1} \cap \cup_{\ell=1}^{K-1} Q_{\ell}\right|, \quad \text { and } \quad \alpha_{2}^{K}=\left|N_{2} \cap \cup_{\ell=1}^{K-1} Q_{\ell}\right|
$$

are the numbers of neighbors of 1 and 2, respectively, for whom $x_{i}$ is iteratively strictly dominant in round $K-1$ or earlier. Hence,

$$
p_{1}<v^{\alpha_{1}^{K}}-\max \left\{v^{d_{1}-\alpha_{1}^{K}}-q_{1}, 0\right\} \quad \text { and } \quad p_{2}<v^{\alpha_{2}^{K}}-\max \left\{v^{d_{2}-\alpha_{2}^{K}}-q_{2}, 0\right\} .
$$

On the other hand, let $p^{\prime}$ be such that $p_{i}^{\prime}=p_{i}$ for $i \neq 2$, and

$$
p_{2}<p_{2}^{\prime}<v^{\alpha_{2}^{K}+1}-\max \left\{v^{d_{2}-\alpha_{2}^{K}-1}-q_{2}, 0\right\} .
$$

Denote by $Q_{k}^{\prime}$ the set of buyers for whom $x_{1}=A$ is an iteratively dominant action in round $k$ under $\left(p^{\prime}, q\right)$ as defined in (5). We then have $Q_{k}^{\prime}=Q_{k}$ for $k=1, \ldots, K-1$ and $Q_{K}^{\prime}=Q_{K} \cup\{1\}$ so that $\alpha_{2}^{K}+1$ of 2 's neighbors have chosen $A$ in round $K$ or earlier. Since

$$
v^{\alpha_{2}^{K}+1}-p_{2}^{\prime}>\max \left\{v^{d_{2}-\alpha_{2}^{K}-1}-q_{2}, 0\right\},
$$

$Q_{K+1}^{\prime}=\{2\}$. Furthermore, since $p_{2}^{\prime}>p_{2}, \pi_{A}\left(p^{\prime}, q, \sigma_{B}\right)>\pi_{A}\left(p, q, \sigma_{B}\right)$. 
Proof of Lemma 4. Fix any relabeling of buyers $i_{1}, \ldots, i_{N}$. Let $s=\left(s_{i}\right)_{i \in I}$ be defined by

$$
s_{i_{1}}=0 \quad \text { and } \quad s_{i_{k}}=\left|N_{i_{k}} \cap\left\{i_{1}, \ldots, i_{k-1}\right\}\right| \text { for } k=2, \ldots, N .
$$

Let $\varepsilon>0$ be given, and define the price vector $p=\left(p_{i}\right)_{i \in I}$ by

$$
p_{i}=\min \left\{v^{s_{i}}-v^{d_{i}-s_{i}}+q_{i}^{*}, v^{s_{i}}\right\}-\varepsilon .
$$

As explained in the text, by offering $p$, seller $A$ makes $x_{i_{1}}=A$ a strictly dominant action for buyer $i_{1}$, and in any subsequent step, $x_{i_{k}}=A$ an iteratively strictly dominant action for buyer $i_{k}$ under $\left(p, q^{*}\right)$. Hence, seller $A^{\prime}$ 's payoff under $\left(p, q^{*}, \sigma\right)$ satisfies

$$
\pi_{A}\left(p, q^{*}, \sigma\right) \geq \sum_{i=1}^{N} \min \left\{v^{s_{i}}-v^{d_{i}-s_{i}}+q_{i}^{*}, v^{s_{i}}\right\}-N \varepsilon .
$$

Since $\varepsilon>0$ and $s \in S$ are arbitrary, if (8) does not hold, then we would have a contradiction

$$
\pi_{A}\left(p, q^{*}, \sigma\right)>\pi_{A}\left(p^{*}, q^{*}, \sigma\right) .
$$

The symmetric argument proves the inequality for seller $B$ 's payoff.

Proof of Proposition 5. We first show that $\left(p^{*}, q^{*}\right)=(0,0)$ is an SPE price. Let $\sigma^{A}$ and $\sigma^{B}$ be the $A$-maximal and $B$-maximal equilibria as defined earlier, and let $\sigma$ be the buyers' strategy profile such that

$$
\sigma(p, q)= \begin{cases}(B, \ldots, B) & \text { if }(p, q)=(0,0), \\ \sigma^{B}(p, q) & \text { if } p \neq 0 \text { and } q=0, \\ \sigma^{A}(p, q) & \text { if } p=0 \text { and } q \neq 0 .\end{cases}
$$

Now consider a deviation from $p^{*}=0$ to $p \neq 0$ by seller $A$. Let $Q_{k}(k=1, \ldots, K)$ be as defined in (5) under $\left(p, q^{*}\right)$. It then follows that

$$
I_{A}\left(\sigma^{A}\left(p, q^{*}\right)\right)=\cup_{k=1}^{K} Q_{k}
$$

for some $K \leq N$. In other words, any buyer attracted by seller $A$ with $p$ must choose $A$ as his iteratively strictly dominant action. Hence, seller $A$ 's payoff under $\left(p, q^{*}, \sigma\right)$ can be written as:

$$
\pi_{A}\left(p, q^{*}, \sigma\right)=\sum_{k=1}^{K} \sum_{i \in Q_{k}} p_{i} .
$$


Now let

$$
\alpha_{i}^{k}=\left|N_{i} \cap \cup_{\ell=1}^{k-1} Q_{\ell}\right|
$$

denote the number of neighbors of buyer $i$ who have already chosen seller $A$ in rounds prior to $k$. If $i \in Q_{k}$, then $x_{i}=A$ must be a dominant action in round $k$ for buyer $i$ so that

$$
v^{\alpha_{i}^{k}}-p_{i}>v^{d_{i}-\alpha_{i}^{k}} \Leftrightarrow p_{i}<v^{\alpha_{i}^{k}}-v^{d_{i}-\alpha_{i}^{k}}
$$

Note now that

$$
\begin{aligned}
& \sum_{k=1}^{K} \sum_{i \in Q_{k}} \alpha_{i}^{k} \\
& =\sum_{k=1}^{K}\left(\# \text { links between } Q_{k} \text { and } \cup_{\ell=1}^{k-1} Q_{\ell}\right) \\
& \leq \# \text { links in the subnetwork } G\left[\cup_{k=1}^{K} Q_{k}\right] \\
& =\frac{1}{2} \sum_{k=1}^{K} \sum_{i \in Q_{k}} d_{i}\left(G\left[\cup_{k=1}^{K} Q_{k}\right]\right) \\
& \leq \frac{1}{2} \sum_{k=1}^{K} \sum_{i \in Q_{k}} d_{i} .
\end{aligned}
$$

Substituting (18), (19) and the linearity of the externalities into (17), we obtain

$$
\begin{aligned}
\pi_{A}\left(p, q^{*}, \sigma\right) & <\sum_{k=1}^{K} \sum_{i \in Q_{k}}\left(v^{\alpha_{i}^{k}}-v^{d_{i}-\alpha_{i}^{k}}\right) \\
& =h \sum_{k=1}^{K} \sum_{i \in Q_{k}}\left(2 \alpha_{i}^{k}-d_{i}\right) \leq 0 .
\end{aligned}
$$

Therefore, the deviation $p$ is not profitable. By the symmetric argument, no deviation $q$ by seller $B$ is profitable either.

Proof of Lemma 6. Note that (12) follows if we show that $d-s$ is not a permutation of $s$ for some $s$ : (11) implies that either $\sum_{i=1}^{N}\left(v^{s_{i}}-v^{d_{i}-s_{i}}\right)>0$ or $<0$. If the latter holds, then let $i_{k}^{\prime}=i_{N-k+1}$ for $k=1, \ldots, N$ and define $t=\left(t_{i}\right)_{i \in I}$ by setting $t_{i_{k}^{\prime}}$ equal to the number of neighbors of $i_{k}^{\prime}$ in $\left\{i_{1}^{\prime}, \ldots, i_{k-1}^{\prime}\right\}$ :

$$
t_{i_{1}^{\prime}}=0 \quad \text { and } \quad t_{i_{k}^{\prime}}=\left|N_{i_{k}^{\prime}} \cap\left\{i_{1}^{\prime}, \ldots, i_{k-1}^{\prime}\right\}\right| \text { for } k=2, \ldots, N \text {. }
$$


Then we can verify that

$$
\sum_{i=1}^{N}\left(v^{t_{i}}-v^{d_{i}-t_{i}}\right)=-\sum_{i=1}^{N}\left(v^{s_{i}}-v^{d_{i}-s_{i}}\right)>0 .
$$

We will consider the following two cases separately.

1) $G$ is not regular.

Take a pair of buyers $i$ and $j$ such that $i$ is adjacent to $j, d_{i}=D$ and $d_{j}<D$, where $D \geq 2$ is the highest degree in $G$. Take another buyer $k$ that is adjacent to $i$ but not to $j$. To see that there exists such a buyer, suppose to the contrary that every buyer $\neq j$ that is adjacent to $i$ is also adjacent to $j$. Then $j$ has at least $D$ neighbors, a contradiction. Let $i_{1}=k, i_{2}=i$ and $i_{3}=j$, and define $i_{4}, \ldots, i_{N} \notin\{i, j, k\}$ arbitrarily. Then

$$
\begin{aligned}
& \left(s_{i_{1}}, s_{i_{2}}, s_{i_{3}}\right)=(0,1,1), \\
& \left(d_{i_{1}}-s_{i_{1}}, d_{i_{2}}-s_{i_{2}}, d_{i_{3}}-s_{i_{3}}\right)=\left(d_{k}, D-1, d_{j}-1\right) .
\end{aligned}
$$

If $s$ is not a permutation of $d-s$, then we are done. Suppose then that $s$ is a permutation of $d-s$, and define $i_{1}^{\prime}=k, i_{2}^{\prime}=j, i_{3}^{\prime}=i$, and $i_{\ell}^{\prime}=i_{\ell}$ for $\ell \geq 4$, and let $t=\left(t_{i}\right)_{i \in I}$ be defined by $(20)$ for these $i_{1}^{\prime}, \ldots, i_{N}^{\prime}$. Then

$$
\begin{aligned}
& \left(t_{i_{1}^{\prime}}, t_{i_{2}^{\prime}}, t_{i_{3}^{\prime}}\right)=(0,0,2) \\
& \left(d_{i_{1}^{\prime}}-t_{i_{1}^{\prime}}, d_{i_{2}^{\prime}}-t_{i_{2}^{\prime}}, d_{i_{3}^{\prime}}-t_{i_{3}^{\prime}}\right)=\left(d_{k}, d_{j}, D-2\right) .
\end{aligned}
$$

Since $i_{\ell}^{\prime}=i_{\ell}$ for $\ell \geq 4$, we have

$$
\begin{aligned}
& \left|\left\{\ell \geq 4: d_{i_{\ell}}-s_{i_{\ell}}=0\right\}\right|=\left|\left\{\ell \geq 4: d_{i_{\ell}^{\prime}}-t_{i_{\ell}^{\prime}}=0\right\}\right|, \\
& \left|\left\{\ell \geq 4: s_{i_{\ell}}=0\right\}\right|=\left|\left\{\ell \geq 4: t_{i_{\ell}^{\prime}}=0\right\}\right| .
\end{aligned}
$$

a) $d_{j}=1$.

In this case,

$$
\left|\left\{\ell \leq 3: d_{i_{\ell}}-s_{i_{\ell}}=0\right\}\right|=\left|\left\{\ell \leq 3: s_{i_{\ell}}=0\right\}\right|=1 .
$$

Hence, since $d-s$ is a permutation of $s$, we must have

$$
\left|\left\{\ell \geq 4: d_{i_{\ell}}-s_{i_{\ell}}=0\right\}\right|=\left|\left\{\ell \geq 4: s_{i_{\ell}}=0\right\}\right| .
$$


It then follows from $(21)$ that

$$
\left|\left\{\ell \geq 4: d_{i_{\ell}^{\prime}}-t_{i_{\ell}^{\prime}}=0\right\}\right|=\left|\left\{\ell \geq 4: t_{i_{\ell}^{\prime}}=0\right\}\right| .
$$

However,

$$
\left|\left\{\ell \leq 3: d_{i_{\ell}^{\prime}}-t_{i_{\ell}^{\prime}}=0\right\}\right| \leq 1<2=\left|\left\{\ell \leq 3: t_{i_{\ell}^{\prime}}=0\right\}\right| .
$$

(22) and (23) together show that $d-t$ cannot be a permutation of $t$.

b) $d_{j} \geq 2$.

In this case, we have $D \geq 3$ since $D>d_{j} \geq 2$, and also

$$
\left|\left\{\ell \leq 3: d_{i_{\ell}}-s_{i_{\ell}}=0\right\}\right|=0<1=\left|\left\{\ell \leq 3: s_{i_{\ell}}=0\right\}\right| .
$$

Hence, since $d-s$ is a permutation of $s$,

$$
\left|\left\{\ell \geq 4: d_{i_{\ell}}-s_{i_{\ell}}=0\right\}\right|=\left|\left\{\ell \geq 4: s_{i_{\ell}}=0\right\}\right|+1 .
$$

It then follows from (21) that

$$
\left|\left\{\ell \geq 4: d_{i_{\ell}^{\prime}}-t_{i_{\ell}^{\prime}}=0\right\}\right|=\left|\left\{\ell \geq 4: t_{i_{\ell}^{\prime}}=0\right\}\right|+1 .
$$

However,

$$
\left|\left\{\ell \leq 3: d_{i_{\ell}^{\prime}}-t_{i_{\ell}^{\prime}}=0\right\}\right|=\left|\left\{\ell \leq 3: t_{i_{\ell}^{\prime}}=0\right\}\right|-2
$$

(24) and (25) together imply that $d-t$ is not a permutation of $t$.

2) $G$ is $r$-regular with $2<r<N-1$.

Since $G$ is connected and not complete, we can take a pair of buyers $i_{1}$ and $i_{2}$ such that $i_{1}$ and $i_{2}$ are adjacent, and take another buyer $i_{3}$ who is adjacent to $i_{2}$ but not to $i_{1}$. To see that this is possible, suppose to the contrary that for any pair of adjacent buyers $i$ and $j$, any buyer $k \neq i$ adjacent to $j$ is also adjacent to $i$. We then show that $G$ must be complete. Take any pair of buyers $i$ and $j$. Since $G$ is connected, there is a path $k_{1}=i \rightarrow k_{2} \rightarrow \cdots \rightarrow k_{m-1} \rightarrow k_{m}=j$. Since $k_{2}$ is adjacent to $i=k_{1}$ and $k_{3}$ is adjacent to $k_{2}, k_{3}$ is adjacent to $i$ as well by the above. Now since $k_{4}$ is adjacent to $k_{3}$, it is also adjacent to $i$. Proceeding the same way, we conclude that $j=k_{m}$ is adjacent to $i=k_{1}$, implying that $G$ is complete. 
We now label buyers other than $\left\{i_{1}, i_{2}, i_{3}\right\}$ as $i_{4}, \ldots, i_{N}$ in an arbitrary manner. For our choice of $i_{1}, i_{2}$ and $i_{3}$, we have

$$
\begin{aligned}
& \left(s_{i_{1}}, s_{i_{2}}, s_{i_{3}}\right)=(0,1,1), \\
& \left(d_{i_{1}}-s_{i_{1}}, d_{i_{2}}-s_{i_{2}}, d_{i_{3}}-s_{i_{3}}\right)=(r, r-1, r-1) .
\end{aligned}
$$

If $d-s$ is a not permutation of $s$, then we are done. Suppose then that $d-s$ is a permutation of $s$. We then must have

$$
\left|\left\{\ell: s_{i_{\ell}}=0\right\}\right|=\left|\left\{\ell: d_{i_{\ell}}-s_{i_{\ell}}=0\right\}\right| .
$$

Let $i_{1}^{\prime}=i_{1}, i_{2}^{\prime}=i_{3}, i_{3}^{\prime}=i_{2}$ and $i_{\ell}^{\prime}=i_{\ell}$ for $\ell \geq 4$, and let $t=\left(t_{i}\right)_{i \in I}$ be defined by (20) for these $i_{1}^{\prime}, \ldots, i_{N}^{\prime}$. Note that

$$
\begin{aligned}
& \left(t_{i_{1}^{\prime}}, t_{i_{2}^{\prime}}, t_{i_{3}^{\prime}}\right)=(0,0,2), \\
& \left(d_{i_{1}^{\prime}}-t_{i_{1}^{\prime}}, d_{i_{2}^{\prime}}-t_{i_{2}^{\prime}}, d_{i_{3}^{\prime}}-t_{i_{3}^{\prime}}\right)=(r, r, r-2) .
\end{aligned}
$$

Since $r>2$, if (26) holds, then the same argument as in the non-regular case shows that

$$
\left|\left\{\ell: t_{i_{\ell}^{\prime}}=0\right\}\right| \neq\left|\left\{\ell: d_{i_{\ell}^{\prime}}-t_{i_{\ell}^{\prime}}=0\right\}\right|,
$$

implying that $d-t$ is not a permutation of $t$.

Proof of Lemma 7. We first show that if $\left(p^{*}, q^{*}, \sigma\right)$ is an SPE, then

$$
\pi_{A}\left(p^{*}, q^{*}, \sigma\right)>\sum_{i=1}^{N} \min \left\{q_{i}^{*}, v^{0}\right\} \quad \text { and } \quad \pi_{B}\left(p^{*}, q^{*}, \sigma\right)>\sum_{i=1}^{N} \min \left\{p_{i}^{*}, v^{0}\right\} .
$$

By Lemma 4, for any $s \in S$, seller $A$ 's payoff under $\left(p^{*}, q^{*}\right)$ satisfies

$$
\pi_{A}\left(p^{*}, q^{*}, \sigma\right) \geq \sum_{i=1}^{N} \min \left\{v^{s_{i}}-v^{d_{i}-s_{i}}+q_{i}^{*}, v^{s_{i}}\right\} .
$$

Rearranging, we get for any $s \in S$,

$$
\begin{aligned}
\pi_{A}\left(p^{*}, q^{*}, \sigma\right) & \geq \sum_{i=1}^{N}\left(v^{s_{i}}-v^{d_{i}-s_{i}}\right)+\sum_{i} \min \left\{q_{i}^{*}, v^{d_{i}-s_{i}}\right\} \\
& \geq \sum_{i=1}^{N}\left(v^{s_{i}}-v^{d_{i}-s_{i}}\right)+\sum_{i=1}^{N} \min \left\{q_{i}^{*}, v^{0}\right\} .
\end{aligned}
$$

When $G$ is neither cyclic or complete, there exists by Lemma 6 an $s \in S$ such that the first term on the right-hand side is $>0$. Hence, the first inequality in (27) must hold. The proof for the second inequality is similar. 
a) If $\min _{i} q_{i}^{*} \geq 0$, then $\pi_{A}\left(p^{*}, q^{*}, \sigma\right)=0 \leq \sum_{i} \min \left\{q_{i}^{*}, v^{0}\right\}$, contradicting (27).

b) If $\max _{i} q_{i}^{*} \leq v^{0}$, then $\pi_{A}\left(p^{*}, q^{*}, \sigma\right) \leq \sum_{i} q_{i}^{*}=\sum_{i} \min \left\{q_{i}^{*}, v^{0}\right\}$, contradicting (27).

c) The inequality $\max _{i} q_{i}^{*}>v^{0}$ follows from (b) above since $I_{B}\left(p^{*}, q^{*}, \sigma\right)=I$ implies $\pi_{A}\left(p^{*}, q^{*}, \sigma\right)=0$ and $0 \leq \pi_{B}\left(p^{*}, q^{*}, \sigma\right)=\sum_{i} q_{i}^{*}$. If $v^{d_{i}}-q_{i}^{*}<v^{0}$ for some $i$, then any $p$ such that $p_{i}=v^{0}-\varepsilon$ and $p_{-i}=0$ for $0<\varepsilon<q_{i}^{*}-v^{d_{i}}+v^{0}$ would induce buyer $i$ to switch to $A$ and hence is a profitable deviation for seller $A$. To see that $v^{D}>2 v^{0}$, note first that $\min _{i}\left(v^{d_{i}}-q_{i}^{*}\right) \geq v^{0}$ in particular implies that $\max _{i} q_{i}^{*} \leq v^{D}-v^{0}$. Hence, if $v^{D} \leq 2 v^{0}$, we have a contradiction to the first statement since $\max _{i} q_{i}^{*} \leq v^{D}-v^{0} \leq v^{0}$.

Proof of Proposition 8. Suppose that $G$ is neither cyclic or complete, and suppose that seller $B$ attracts all the buyers in an $\operatorname{SPE}\left(p^{*}, q^{*}, \sigma\right)$ such that $q_{1}^{*}=\cdots=$ $q_{N}^{*}$. Then since $\pi_{A}\left(p^{*}, q^{*}, \sigma\right)=0$, Lemma $7(1)$ implies that $q_{1}^{*}=\cdots=q_{N}^{*}=$ $\min _{i} q_{i}^{*}<0$. Then, however, $\pi_{B}\left(p^{*}, q^{*}, \sigma\right)<0$, a contradiction.

Proof of Proposition 9 It suffices to show that $\left(p^{*}, q^{*}\right)=(0,0)$ coupled with the following strategy profile $\sigma$ of the buyers is an SPE in each class of networks:

$$
\sigma(p, q)= \begin{cases}(B, \ldots, B) & \text { if }(p, q)=\left(p^{*}, q^{*}\right), \\ \sigma^{A}(p, q) & \text { if } p=p^{*} \text { and } q \neq q^{*} \\ \sigma^{B}(p, q) & \text { if } p \neq p^{*} \text { and } q q^{*}\end{cases}
$$

In other words, all buyers choose $B$ under $\left(p^{*}, q^{*}\right)=(0,0)$, and when one of the firms deviates to a non-zero price vector, the buyers coordinate on the NE which is least favorable to the deviating seller. In what follows, we show that seller $A$ has no incentive to deviate. A symmetric argument shows that seller $B$ has no incentive to deviate.

1) $G$ is a cycle.

Suppose that seller $A$ deviates to $p \neq p^{*}$. Let $Q_{k}$ be as defined in (5) under $\left(p, q^{*}\right)$. Since

$$
I_{A}\left(\sigma\left(p, q^{*}\right)\right)=\cup_{k=1}^{K} Q_{k}
$$

for some $K \leq N$, if $\sigma_{i}\left(p, q^{*}\right)=A$, then $i \in Q_{k}$ for some $k \leq K$. Recall that $N_{i}$ is the set of neighbors of $i$ in $G$, and that $d_{i}=\left|N_{i}\right|=2$ since $G$ is cyclic. Let

$$
\alpha_{i}^{k}=\left|N_{i} \cap \cup_{\ell=1}^{k-1} Q_{\ell}\right| \in\{0,1,2\}
$$


denote the number of $i$ 's neighbors who have chosen $A$ in rounds prior to $k$. If $i \in Q_{k}$, then $x_{i}=A$ is a dominant action in round $k$ for buyer $i$ so that

$$
v^{\alpha_{i}^{k}}-p_{i}>v^{2-\alpha_{i}^{k}} \Leftrightarrow p_{i}<v^{\alpha_{i}^{k}}-v^{2-\alpha_{i}^{k}} .
$$

In particular, buyer $i$ is attracted by seller $A$ in round 1 if $p_{i}<v^{0}-v^{2}$, and attracted by $A$ in round $k>1$ either if (i) $p_{i}<0$ and exactly one of his two neighbors has already chosen $A\left(\alpha_{i}^{k}=1\right)$, or (ii) $p_{i}<v^{2}-v^{0}$ and both his neighbors have already chosen $A\left(\alpha_{i}^{k}=2\right)$. Note also that only in round 1 does any buyer choose $A$ when neither of his neighbors have already chosen $A$.

Seller $A$ 's payoff under $\left(p, q^{*}, \sigma\right)$ hence satisfies

$$
\begin{aligned}
\pi_{A}\left(p, q^{*}, \sigma\right) & =\sum_{k=1}^{K} \sum_{i \in Q_{k}} p_{i} \\
& <\left|Q_{1}\right|\left(v^{0}-v^{2}\right)+\left(v^{2}-v^{0}\right) \sum_{k=2}^{K}\left|\left\{i \in I \backslash\left(\cup_{\ell=1}^{k-1} Q_{\ell}\right): \alpha_{i}^{k}=2\right\}\right| .
\end{aligned}
$$

Since no buyer chooses $A$ in round $k \geq 2$ if neither of his neighbors has already chosen $A$, the number of components in $G\left[\cup_{\ell=1}^{k-1} Q_{\ell}\right]$ is less than or equal to that in $G\left[Q_{1}\right]$ for any $k$. It follows that

$$
\sum_{k=2}^{K}\left|\left\{i \in I \backslash\left(\cup_{\ell=1}^{k-1} Q_{\ell}\right): \alpha_{i}^{k}=2\right\}\right| \leq\left|Q_{1}\right| .
$$

We can therefore conclude that $\pi_{A}\left(p, q^{*}, \sigma\right) \leq 0$ and hence that $p$ is not a profitably deviation.

2) $G$ is complete.

Define $Q_{k}(k=1, \ldots, K)$ as above. Since $G$ is complete, for any buyer $i$, the number $\alpha_{i}^{k}$ of $i$ 's neighbors who have chosen $A$ equals the number $\alpha_{k}$ of buyers who have chosen $A$ in rounds $1, \ldots, k-1$ :

$$
\alpha_{i}^{k}=\left|N_{i} \cap \cup_{\ell=1}^{k-1} Q_{\ell}\right|=\sum_{\ell=1}^{k-1}\left|Q_{\ell}\right| \equiv \alpha_{k} .
$$

Furthermore, by Proposition 3, we only need consider $p$ such that each $Q_{k}$ contains a single buyer. (If $Q_{k}$ contains two or more buyers, then since $G$ is complete, those buyers are adjacent.) Hence, without loss of generality, $Q_{k}=\{k\}$ for each $k=1, \ldots, N$. For $k=1, \ldots, K$, we also have

$$
p_{k}<v^{\alpha_{k}}-v^{N-1-\alpha_{k}} .
$$


Seller $A$ 's payoff under $\left(p, q^{*}, \sigma\right)$ hence satisfies

$$
\pi_{A}\left(p, q^{*}, \sigma\right)=\sum_{k=1}^{N} \sum_{k=1}^{N} p_{k}<\sum_{k=1}^{K}\left(v^{\alpha_{k}}-v^{N-1-\alpha_{k}}\right) .
$$

It is then straightforward to verify that the right-hand side equals zero. Hence, seller $A$ has no profitable deviation.

Proof of Proposition 10. We will construct an $\operatorname{SPE}\left(p^{*}, q^{*}, \sigma\right)$ in which seller $B$ captures all the buyers: $I_{B}\left(\sigma\left(p^{*}, q^{*}\right)\right)=I$. Let the buyer set be partitioned into $I_{1}$ and $I_{2}$ so that links exist only between $I_{1}$ and $I_{2}$. Suppose without loss of generality that

$$
\sum_{i \in I_{1}}\left(v^{d_{i}}-v^{0}\right)-\sum_{i \in I_{2}}\left(v^{d_{i}}-v^{0}\right) \geq 0
$$

Let

$$
p_{i}^{*}=q_{i}^{*}= \begin{cases}v^{d_{i}}-v^{0} & \text { if } i \in I_{1}, \\ v^{0}-v^{d_{i}} & \text { if } i \in I_{2},\end{cases}
$$

and

$$
\sigma(p, q)= \begin{cases}\sigma^{B}(p, q) & \text { if } q=q^{*} \\ \sigma^{A}(p, q) & \text { otherwise. }\end{cases}
$$

By $(30)$, seller $B$ 's payoff under $\left(p^{*}, q^{*}, \sigma\right)$ is non-negative:

$$
\pi_{B}\left(p^{*}, q^{*}, \sigma\right)=\sum_{i \in I} q_{i}^{*} \geq 0 .
$$

By the definition of the $B$-maximal NE, if seller $A$ deviates to $p$, then the set of buyers he captures equals $I_{A}\left(\sigma\left(p, q^{*}\right)\right)=\cup_{k=1}^{K} Q_{k}$, where $Q_{k}$ is the set of buyers $i$ for whom $x_{i}=A$ is a strictly dominant strategy in round $k$ under $\left(p, q^{*}\right)$ as defined in $(5)$.

Suppose first that $Q_{1} \subset I_{1}$. we then have

$$
\sum_{i \in Q_{1}} p_{i}<\sum_{i \in Q_{1}} \min \left\{v^{0}-v^{d_{i}}+q_{i}^{*}, v^{0}\right\}=0
$$

Therefore, no $p$ such that $K=1$ and $Q_{1} \subset I_{1}$ under $\left(p, q^{*}\right)$ is profitable. Since $q_{i}^{*}<0$ for $i \in I_{2}$, it is clear that no $p$ such that $K=1$ under $\left(p, q^{*}\right)$ is profitable either. 
Suppose next that $K=2$ and that $Q_{1} \subset I_{1}$. Then $Q_{2} \cap I_{1}=\emptyset$ since in round 2 , every buyer $i \in Q_{1}$ must be adjacent to some buyer in $Q_{1} \subset I_{1}$. (Otherwise, $i$ would have been in $Q_{1}$.) It follows that $q_{i}^{*}<0$ for each $i \in Q_{2}$, and hence that

$$
\begin{aligned}
\sum_{i \in Q_{2}} p_{i} & <\sum_{i \in Q_{2}} \min \left\{v^{\alpha_{i}^{2}}-v^{d_{i}-\alpha_{i}^{2}}+q_{i}^{*}, v^{\alpha_{i}^{2}}\right\} \\
& =\sum_{i \in Q_{2}}\left(v^{\alpha_{i}^{2}}-v^{d_{i}-\alpha_{i}^{2}}-v^{d_{i}}+v^{0}\right) \leq 0,
\end{aligned}
$$

where $\alpha_{i}^{2}=\left|N_{i} \cap Q_{1}\right|$ is the number of $i$ 's neighbors in $Q_{1}$. Therefore, no deviation $p$ is profitable if $K=2, Q_{1} \subset I_{1}$ and $Q_{2} \subset I_{2}$ under $\left(p, q^{*}\right)$. It is then also clear that no deviation $p$ is profitable if $K=2$ and $Q_{2} \subset I_{2}$.

We next show that no deviation $p$ is profitable if $K \geq 2$ and $Q_{k} \cap I_{1} \neq \emptyset$ for some $k \geq 2$. Together with the above observations, this would imply that no deviation $p$ is profitable if $K=2$. Furthermore, if $K \geq 3$, then it must be the case that either $Q_{2} \cap I_{1} \neq \emptyset$ or $Q_{3} \cap I_{1} \neq \emptyset$ since $G$ is bipartite, and since every $i \in Q_{3}$ is adjacent to some buyer in $Q_{2}$. It would hence follow that no deviation $p$ is profitable.

Let $j \in Q_{k} \cap I_{1}$ for some $k \geq 2$. Then

$$
\begin{aligned}
\pi_{A}\left(p, q^{*}, \sigma\right) & =\sum_{\ell=1}^{K} \sum_{i \in Q_{\ell}} p_{i} \\
& <\sum_{\ell=1}^{K} \sum_{i \in Q_{\ell}} \min \left\{v^{\alpha_{i}^{\ell}}-v^{d_{i}-\alpha_{i}^{\ell}}+q_{i}^{*}, v^{\alpha_{i}^{\ell}}\right\} \\
& \leq \sum_{\ell=1}^{K} \sum_{i \in Q_{\ell}}\left(v^{\alpha_{i}^{\ell}}-v^{d_{i}-\alpha_{i}^{\ell}}+q_{i}^{*}\right)+v^{\alpha_{j}^{k}}-\left(v^{d_{j}}-v^{0}+q_{j}\right),
\end{aligned}
$$

where

$$
\alpha_{i}^{\ell}=\left|N_{i} \cap\left(\cup_{\kappa=1}^{\ell-1} Q_{\kappa}\right)\right|
$$

is the number of $i$ 's neighbors who have chosen $A$ prior to round $\ell$. We now use approximate linearity to evaluate the right-hand side of (31) term by term. First, since $q_{j}^{*}=v^{d_{j}}-v^{0}$,

$$
v^{\alpha_{j}^{k}}-\left(v^{d_{j}}-v^{0}+q_{j}^{*}\right) \leq-v^{d_{j}}+2 v^{0}<-h d_{j}+3 \varepsilon .
$$


Observe next that

$$
\begin{aligned}
& \sum_{\ell=1}^{K} \sum_{i \in Q_{\ell}}\left\{v^{\alpha_{i}^{\ell}}-v^{d_{i}-\alpha_{i}^{\ell}}\right\} \\
& =\sum_{\ell=1}^{K} \sum_{i \in Q_{\ell}}\left[\left\{v^{\alpha_{i}^{\ell}}-\alpha_{i}^{\ell} h\right\}-\left\{v^{d_{i}-\alpha_{i}^{\ell}}-\left(d_{i}-\alpha_{i}^{\ell}\right) h\right\}\right] \\
& -\sum_{\ell=1}^{K} \sum_{i \in Q_{\ell}}\left(\left(d_{i}-\alpha_{i}^{\ell}\right)-\alpha_{i}^{\ell}\right) h \\
& \leq 2 \varepsilon\left|\cup_{\ell=1}^{K} Q_{\ell}\right|-h m,
\end{aligned}
$$

where

$$
m=\text { \#links between } \cup_{\ell=1}^{K} Q_{\ell} \text { and } I \backslash\left(\cup_{\ell=1}^{K} Q_{\ell}\right) .
$$

Observe finally that

$$
\begin{aligned}
& \sum_{\ell=1}^{K} \sum_{i \in Q_{\ell}} q_{i}^{*} \\
& =\sum_{\ell=1}^{K} \sum_{i \in Q_{\ell} \cap I_{1}}\left(v^{d_{i}}-v^{0}\right)-\sum_{\ell=1}^{K} \sum_{i \in Q_{\ell} \cap I_{2}}\left(v^{d_{i}}-v^{0}\right) \\
& =\sum_{\ell=1}^{K} \sum_{i \in Q_{\ell} \cap I_{1}}\left(v^{d_{i}}-v^{0}-d_{i} h\right)-\sum_{\ell=1}^{K} \sum_{i \in Q_{\ell} \cap I_{2}}\left(v^{d_{i}}-v^{0}-d_{i} h\right) \\
& +h \sum_{\ell=1}^{K} \sum_{i \in Q_{\ell} \cap I_{1}} d_{i}-h \sum_{\ell=1}^{K} \sum_{i \in Q_{\ell} \cap I_{2}} d_{i} .
\end{aligned}
$$

Since the externalities are $\varepsilon$-close to linear,

$$
\sum_{\ell=1}^{K} \sum_{i \in Q_{\ell} \cap I_{1}}\left(v^{d_{i}}-v^{0}-d_{i} h\right)-\sum_{\ell=1}^{K} \sum_{i \in Q_{\ell} \cap I_{2}}\left(v^{d_{i}}-v^{0}-d_{i} h\right) \leq 2 \varepsilon\left|\cup_{\ell=1}^{K} Q_{\ell}\right| .
$$

We also have

$$
\begin{aligned}
\sum_{\ell=1}^{K} \sum_{i \in Q_{\ell} \cap I_{1}} d_{i} & =\text { \#links between } \cup_{\ell=1}^{K} Q_{\ell} \cap I_{1} \text { and } I_{2} \\
\sum_{\ell=1}^{K} \sum_{i \in Q_{\ell} \cap I_{2}} d_{i} & =\text { \#links between } \cup_{\ell=1}^{K} Q_{\ell} \cap I_{2} \text { and } I_{1} \\
& \geq \# \text { links between } \cup_{\ell=1}^{K} Q_{\ell} \cap I_{2} \text { and } \cup_{\ell=1}^{K} Q_{\ell} \cap I_{1}
\end{aligned}
$$


so that

$$
\begin{aligned}
& \sum_{\ell=1}^{K} \sum_{i \in Q_{\ell} \cap I_{1}} d_{i}-\sum_{\ell=1}^{K} \sum_{i \in Q_{\ell} \cap I_{2}} d_{i} \\
& \leq \# \text { links between } \cup_{\ell=1}^{K} Q_{\ell} \text { and } I_{2} \backslash\left(\cup_{\ell=1}^{K} Q_{\ell}\right) \\
& \leq \# \text { links between } \cup_{\ell=1}^{K} Q_{\ell} \text { and } I \backslash\left(\cup_{\ell=1}^{K} Q_{\ell}\right) \\
& =m .
\end{aligned}
$$

Substituting (35) and (36) into (34), we obtain

$$
\sum_{\ell=1}^{K} \sum_{i \in Q_{\ell}} q_{i}^{*} \leq 2 \varepsilon\left|\cup_{\ell=1}^{K} Q_{\ell}\right|+h m .
$$

Substituting (32), (33) and (37) into (31), we see that

$$
\begin{aligned}
\pi_{A}\left(p, q^{*}, \sigma\right) & <2 \varepsilon\left|\cup_{\ell=1}^{K} Q_{\ell}\right|-h m+2 \varepsilon\left|\cup_{\ell=1}^{K} Q_{\ell}\right|+h m-h d_{j}+3 \varepsilon \\
& =\varepsilon\left(4\left|\cup_{\ell=1}^{K} Q_{\ell}\right|+3\right)-h d_{j} \\
& \leq \varepsilon(4 N+3)-h d_{j} .
\end{aligned}
$$

Hence, if we set $\bar{\varepsilon}=h /(4 N+3)$, then $\pi_{A}\left(p, q^{*}, \sigma\right)<0$ when $\varepsilon<\bar{\varepsilon}$.

Proof of Proposition 12. Let

$$
\delta=\max _{s \in S} \sum_{i=1}^{N}\left(v^{s_{i}}-v^{d_{i}-s_{i}}\right) .
$$

When the externalities are $\varepsilon$-close to $h$-linear,

$$
\begin{aligned}
\sum_{i=1}^{N}\left(v^{s_{i}}-v^{d_{i}-s_{i}}\right) & =\sum_{i=1}^{N}\left\{\left(v^{s_{i}}-s_{i} h\right)-\left(v^{d_{i}-s_{i}}-\left(d_{i}-s_{i}\right) h\right)-h\left(\left(d_{i}-s_{i}\right)-s_{i}\right)\right\} \\
& <2 N \varepsilon
\end{aligned}
$$

and hence

$$
\delta<2 N \varepsilon
$$

Since $G$ is separable, let $\left(I_{1}, I_{2}\right)$ be the partition of the buyer set $I$, and let $i_{A} \in I_{1}$ and $i_{B} \in I_{2}$ be such that

$$
\left|N_{i_{A}} \cap I_{1}\right|>\left|N_{i_{A}} \cap I_{2}\right| \text { and }\left|N_{i_{B}} \cap I_{2}\right|>\left|N_{i_{B}} \cap I_{1}\right| \text {. }
$$


We specify $\left(p^{*}, q^{*}, \sigma\right)$ as follows:

$$
\left(p_{i}^{*}, q_{i}^{*}\right)= \begin{cases}(\delta,-\delta) & \text { if } i=i_{A} \\ (-\delta, \delta) & \text { if } i=i_{B} \\ (0,0) & \text { otherwise }\end{cases}
$$

and

$$
\sigma(p, q)= \begin{cases}(\underbrace{A, \ldots, A}_{I_{1}}, \underbrace{B, \ldots, B}_{I_{2}}) & \text { if }(p, q)=\left(p^{*}, q^{*}\right), \\ \sigma^{B}(p, q) & \text { if } p \neq p^{*}, \\ \sigma^{A}(p, q) & \text { if } p=p^{*} \text { and } q \neq q^{*} .\end{cases}
$$

Note that $\pi_{A}\left(p^{*}, q^{*}, \sigma\right)=\pi_{B}\left(p^{*}, q^{*}, \sigma\right)=\delta$.

We first show that the buyers' action profile following $\left(p^{*}, q^{*}\right)$ is a NE. If $i \in$ $I_{1} \backslash\left\{i_{A}\right\}$, then $x_{i}=A$ is a best response since

$$
v^{\left|N_{i} \cap I_{1}\right|}-p_{i}=v^{\left|N_{i} \cap I_{1}\right|} \geq v^{\left|N_{i} \cap I_{2}\right|}=v^{\left|N_{i} \cap I_{2}\right|}-q_{i} .
$$

If $i=i_{A}$, then $\left|N_{i} \cap I_{1}\right|>\left|N_{i} \cap I_{2}\right|$ so that

$$
\begin{aligned}
& v^{\left|N_{i} \cap I_{1}\right|}-v^{\left|N_{i} \cap I_{2}\right|} \\
& =\left(v^{\left|N_{i} \cap I_{1}\right|}-h\left|N_{i} \cap I_{1}\right|\right)-\left(v^{\left|N_{i} \cap I_{2}\right|}-h\left|N_{i} \cap I_{2}\right|\right)+h\left\{\left|N_{i} \cap I_{1}\right|-\left|N_{i} \cap I_{2}\right|\right\} \\
& \geq h-2 \varepsilon .
\end{aligned}
$$

Hence, if we take

$$
\bar{\varepsilon}=\frac{h}{2(2 N+1)},
$$

then for any $\varepsilon<\bar{\varepsilon}$, (38) implies that

$$
v^{\left|N_{i} \cap I_{1}\right|}-p_{i}=v^{\left|N_{i} \cap I_{1}\right|}-\delta>v^{\left|N_{i} \cap I_{2}\right|}+\delta=v^{\left|N_{i} \cap I_{2}\right|}-q_{i} .
$$

The symmetric argument shows that $x_{i}=B$ is a best response for each $i \in I_{2}$ following $\left(p^{*}, q^{*}\right)$.

We will next show that seller $A$ has no profitable deviation. Let $p$ be any deviation by seller $A$, and denote by $Q_{k}$ the set of buyers who will choose $A$ as an iteratively dominant action in round $k$ under $\left(p, q^{*}\right)$ as defined in (5). Since the buyers play $\sigma^{B}$ following $\left(p, q^{*}\right)$, buyer $i$ will choose $A$ only if $x_{i}=A$ is iteratively dominant: $i \in \cup_{k=1}^{K} Q_{k}$. By Proposition 3, we may assume that no buyers in $Q_{k}$ are adjacent. 
If $i \in Q_{k}$, then

$$
p_{i}<\min \left\{v^{\alpha_{i}^{k}}-v^{d_{i}-\alpha_{i}^{k}}+q_{i}^{*}, v^{\alpha_{i}^{k}}\right\} \leq v^{\alpha_{i}^{k}}-v^{d_{i}-\alpha_{i}^{k}}+q_{i}^{*},
$$

where

$$
\alpha_{i}^{k}=\left|N_{i} \cap\left(\cup_{\kappa=1}^{k-1} Q_{\kappa}\right)\right|
$$

is the number of $i$ 's neighbors who have chosen $A$ prior to round $k$. Suppose first that $\cup_{k=1}^{K} Q_{k} \subsetneq I$. Since the externalities are $\varepsilon$-close to $h$-linear, we have

$$
\begin{aligned}
& v^{\alpha_{i}^{k}}-v^{d_{i}-\alpha_{i}^{k}} \\
& =\left(v^{\alpha_{i}^{k}}-\alpha_{i}^{k} h\right)-\left(v^{d_{i}-\alpha_{i}^{k}}-\left(d_{i}-\alpha_{i}^{k}\right) h\right)-\left(\left(d_{i}-\alpha_{i}^{k}\right)-\alpha_{i}^{k}\right) h \\
& <2 \varepsilon-\left(\left(d_{i}-\alpha_{i}^{k}\right)-\alpha_{i}^{k}\right) h .
\end{aligned}
$$

Hence,

$$
\begin{aligned}
\pi_{A}\left(p, q^{*}, \sigma\right) & =\sum_{k=1}^{K} \sum_{i \in Q_{k}} p_{i} \\
& \leq \sum_{k=1}^{K} \sum_{i \in Q_{k}}\left(v^{\alpha_{i}^{k}}-v^{d_{i}-\alpha_{i}^{k}}+q_{i}^{*}\right) \\
& <\sum_{k=1}^{K} \sum_{i \in Q_{k}}\left\{2 \varepsilon-\left(\left(d_{i}-\alpha_{i}^{k}\right)-\alpha_{i}^{k}\right) h\right\}+\sum_{k=1}^{K} \sum_{i \in Q_{k}} q_{i}^{*} \\
& =2 \varepsilon\left|\cup_{k=1}^{K} Q_{k}\right|-h \sum_{k=1}^{K} \sum_{i \in Q_{k}}\left(\left(d_{i}-\alpha_{i}^{k}\right)-\alpha_{i}^{k}\right)+\sum_{k=1}^{K} \sum_{i \in Q_{k}} q_{i}^{*} .
\end{aligned}
$$

Since $\cup_{k=1}^{K} Q_{k} \subsetneq I$ by assumption and since $G$ is connected,

$$
\sum_{k=1}^{K} \sum_{i \in Q_{k}}\left(\left(d_{i}-\alpha_{i}^{k}\right)-\alpha_{i}^{k}\right)=\text { \#links between } \cup_{k=1}^{K} Q_{k} \text { and } I \backslash \cup_{k=1}^{K} Q_{k} \geq 1 \text {. }
$$

It hence follows from (38) that

$$
\pi_{A}\left(p, q^{*}, \sigma\right)<2 \varepsilon\left|\cup_{k=1}^{K} Q_{k}\right|-h+2 N \varepsilon<4 N \varepsilon-h,
$$

which is $<0$ for $\varepsilon<\bar{\varepsilon}$ when $\bar{\varepsilon}$ is given in (39).

Suppose next that $\cup_{k=1}^{K} Q_{k}=I$. In this case, $\sum_{k=1}^{K} \sum_{i \in Q_{k}} q_{i}^{*}=0$. Hence the definition of $\delta$ implies that

$$
\pi_{A}\left(p, q^{*}, \sigma\right)=\sum_{k=1}^{K} \sum_{i \in Q_{k}} p_{i} \leq \sum_{k=1}^{K} \sum_{i \in Q_{k}}\left(v^{\alpha_{i}^{k}}-v^{d_{i}-\alpha_{i}^{k}}+q_{i}^{*}\right) \leq \delta=\pi_{A}\left(p^{*}, q^{*}, \sigma\right) .
$$

In either case, hence, the deviation $p$ is not profitable. 


\section{Appendix II: Strong Equilibrium in the Buyers' Game}

Our discussion in the text has placed no restriction on the buyers' strategies other than that implied by a Nash equilibrium. In this Appendix, we ask what happens when the buyers actively coordinate their actions. While there can be many different formulations of action coordination, one simple and extreme way is to suppose that any subset of the buyers may choose a joint deviation whenever that yields each one of them a strictly higher payoff than adhering to the proposed action profile. In other words, we will require that in each subgame, the buyers' action profile constitutes a strong Nash equilibrium. ${ }^{9}$ We find that the marginal cost pricing is not consistent with a strong Nash equilibrium even if the network is complete or cyclic.

Formally, the buyers action profile $x^{*}$ is a strong Nash equilibrium (strong NE) if for any nonempty subset $J \subset I$ of buyers, and for any $x_{J}$,

$$
u_{i}\left(x^{*}\right) \geq u_{i}\left(x_{J}, x_{-J}^{*}\right) \text { for some } i \in J \text {. }
$$

In other words, an action profile is a strong NE if, whenever a coalition of buyers contemplate a joint deviation, there is a member in the coalition who cannot strictly benefit from the deviation. If $x^{*}$ is a strong NE, then it is clearly a NE. Note also that $x^{*}$ is a strong NE if and only if (40) holds for any non-empty $J \subset I$ and any $x_{J}$ such that $x_{j} \neq x_{j}^{*}$ for every $j \in J .^{10}$

A strong NE $x$ of the buyers' subgame is $A$-maximal if for any strong NE $y$, $y_{i}=A$ implies $x_{i}=A$, and $B$-maximal if $y_{i}=B$ implies $x_{i}=B$. We can find these maximal strong NE using the iteration procedure similar to that used to find the $A$-maximal and $B$-maximal NE.

For any action profiles $x$ and $y$, identify $u_{i}\left(x_{\emptyset}, y\right)$ with $u_{i}(y)$. Let $T_{0}=\emptyset$, and define the subsets of buyers $T_{k}, P_{k}, Q_{k}, R_{k}, Y_{k}$ and $Z_{k}(n=1,2, \ldots)$ recursively as follows.

\footnotetext{
${ }^{9}$ By the property of the payoff functions of the buyers' game, we can verify that any strong Nash equilibrium is a coalition-proof Nash equilibrium in the sense of Bernheim et al. (1987).

${ }^{10}$ To see this, suppose that $x^{*}$ is not a strong NE. Then there exist $J \neq \emptyset$ and $x_{J}$ such that $u_{j}\left(x^{*}\right)<u_{j}\left(x_{J}, x_{-J}^{*}\right)$ for every $j \in J$. Then $J^{\prime} \equiv\left\{j \in J: x_{j} \neq x_{j}^{*}\right\} \neq \emptyset$. Moreover,

$$
u_{j}\left(x_{J^{\prime}}, x_{-J^{\prime}}^{*}\right)=u_{j}\left(x_{J^{\prime}}, x_{J \backslash J^{\prime}}, x_{-J}\right)=u_{j}\left(x_{J}, x_{-J}^{*}\right)>u_{j}\left(x^{*}\right)
$$

for every $j \in J^{\prime}$ since $j \in J \backslash J^{\prime}$ implies $x_{j}=x_{j}^{*}$. Hence, the coalition $J^{\prime}$ also has a profitable joint deviation such that $x_{j} \neq x_{j}^{*}$ for every $j \in J^{\prime}$.
} 
For $k=0,1,2, \ldots$, define $Y_{k+1} \subset I \backslash T_{k}$ to be the maximal set such that

$$
Y_{k+1}=\left\{i \in I \backslash T_{k}: u_{i}\left(x_{T_{k}}^{*}, x_{Y_{k+1}}=(A, \ldots, A), x_{-T_{k}-Y_{k+1}}=(\emptyset, \ldots, \emptyset)\right) \geq 0\right\} .
$$

$Y_{k+1}$ is the set of buyers not in $T_{k}$ who, given $x_{T_{k}}^{*}$, can collectively choose $A$ and enjoy non-negative payoffs from it. ${ }^{11}$ If there is no such set, let $Y_{k+1}=\emptyset$. Likewise, define $Z_{k+1} \subset I \backslash T_{k}$ to be the maximal set such that

$$
Z_{k+1}=\left\{i \in I \backslash T_{k}: u_{i}\left(x_{T_{k}}^{*}, x_{Z_{k+1}}=(B, \ldots, B), x_{-T_{k}-Z_{k+1}}=(\emptyset, \ldots, \emptyset)\right) \geq 0\right\} .
$$

If there is no such set, then let $Z_{k+1}=\emptyset$. Let also $R_{k+1}$ be defined by

$$
R_{k+1}=\left(I \backslash T_{k}\right) \backslash\left(Y_{k+1} \cup Z_{k+1}\right) .
$$

As before, $R_{k+1}$ is the set of buyers $i$ for whom $x_{i}=\emptyset$ is iteratively strictly dominant given $x_{T_{k}}^{*}$. Now define $P_{k+1} \subset I \backslash T_{k}$ to be the maximal set such that

$$
\begin{aligned}
P_{k+1}=\left\{i \in I \backslash T_{k}:\right. & u_{i}\left(x_{T_{k}}^{*}, x_{P_{k+1}}=(B, \ldots, B), x_{-T_{k}-P_{k+1}}=(\emptyset, \ldots, \emptyset)\right) \\
> & \left.u_{i}\left(x_{T_{k}}^{*}, x_{Y_{k+1}}=(A, \ldots, A), x_{-T_{k}-Y_{k+1}}=(\emptyset, \ldots, \emptyset)\right)\right\} .
\end{aligned}
$$

If there exists no such set, then let $P_{k+1}=\emptyset . P_{k+1}$ is the set of buyers whose payoffs from collectively choosing $x_{i}=B$ are strictly higher than those from the maximal coordination on $A$ or from $\emptyset$. Likewise, define $Q_{k+1} \subset I \backslash T_{k}$ to be the maximal set of buyers whose payoffs from collectively choosing $x_{i}=A$ are strictly higher than those from the maximal coordination on $B$ or from $\emptyset$ :

$$
\begin{aligned}
Q_{k+1}=\left\{i \in I \backslash T_{k}:\right. & u_{i}\left(x_{T_{k}}^{*}, x_{Q_{k+1}}=(A, \ldots, A), x_{-T_{k}-Q_{k+1}}=(\emptyset, \ldots, \emptyset)\right) \\
> & \left.u_{i}\left(x_{T_{k}}^{*}, x_{Z_{k+1}}=(B, \ldots, B), x_{-T_{k}-Z_{k+1}}=(\emptyset, \ldots, \emptyset)\right)\right\} .
\end{aligned}
$$

Again, if there exists no such set, then let $Q_{k+1}=\emptyset$.

1) If $P_{k+1}=Q_{k+1}=R_{k+1}=\emptyset$, then let $k=K$ and stop.

2) Otherwise, let

$$
T_{k+1}=T_{k} \cup\left(P_{k+1} \cup Q_{k+1} \cup R_{k+1}\right),
$$

and

$$
x_{i}^{*}= \begin{cases}B & \text { if } i \in P_{k+1}, \\ A & \text { if } i \in Q_{k+1}, \\ \emptyset & \text { if } i \in R_{k+1} .\end{cases}
$$

\footnotetext{
${ }^{11}$ With the possible difference in $T_{k}$ and $x_{T_{k}}$, hence, the definition of $Y_{k+1}$ is the same as in (2).
} 
If $T_{k+1}=I$, then set $K=k+1$ and stop. Otherwise, increase $k$ by one and start over.

Given that the above process starts over only when there is a buyer who has a joint dominant action, the maximal number of iteration $K \leq N$.

Proposition 14 Let $x^{A}$ and $x^{B}$ be defined by

$$
\begin{aligned}
& x^{A}=\left(x_{T_{K}}^{*}, x_{Y_{K+1}}=(A, \ldots, A), x_{-T_{K}-Y_{K+1}}=(\emptyset, \ldots, \emptyset)\right), \quad \text { and } \\
& x^{B}=\left(x_{T_{K}}^{*}, x_{Z_{K+1}}=(B, \ldots, B), x_{-T_{K}-Z_{K+1}}=(\emptyset, \ldots, \emptyset)\right) .
\end{aligned}
$$

Then $x^{A}$ and $x^{B}$ are the $A$-maximal and B-maximal strong $N E$, respectively.

$\left(p^{*}, q^{*}, \sigma\right)$ is a strong SPE if for every $(p, q), \sigma(p, q)$ is a strong NE of the buyers' subgame, and $\pi_{A}\left(p^{*}, q^{*}, \sigma\right) \geq \pi_{A}\left(p, q^{*}, \sigma\right)$ and $\pi_{B}\left(p^{*}, q^{*}, \sigma\right) \geq \pi_{B}\left(p^{*}, q, \sigma\right)$ for every $p$ and $q$.

Proposition 15 Let a buyer network $G$ be given and the externalities $v=\left(v^{0}, \ldots, v^{D}\right)$ satisfy (11). There exists no buyers' strategy profile $\sigma$ such that for $p^{*}=q^{*}=0$ $\left(p^{*}, q^{*}, \sigma\right)$ is a strong SPE.

Proof of Proposition 14. We show that $x^{A}$ is an $A$-maximal strong NE. The symmetric argument shows that $x^{B}$ is a $B$-maximal strong NE. We begin by making some preliminary observations as follows:

Lemma 16 a) $u_{i}\left(x^{A}\right) \geq 0$ for every $i$.

b) For any $k=1, \ldots, K$,

$$
\left\{i \in I \backslash T_{k}: x_{i}^{A}=A\right\} \subset Y_{k+1}, \quad \text { and } \quad\left\{i \in I \backslash T_{k}: x_{i}^{A}=B\right\} \subset Z_{k+1} .
$$

c) For any $k, J \subset I \backslash T_{k}$ and $x_{J}$, if $u_{i}\left(x_{J}, x_{-J}^{A}\right) \geq 0$ for every $i \in J$, then

$$
\left\{i \in J: x_{i}=A\right\} \subset Y_{k+1} \quad \text { and } \quad\left\{i \in J: x_{i}=B\right\} \subset Z_{k+1} \text {. }
$$

In particular, for any $y_{-T_{k}}$ such that $u_{i}\left(y_{-T_{k}}, x_{T_{k}}^{A}\right) \geq 0$ for every $i \in I \backslash T_{k}$,

$$
\left\{i \in I \backslash T_{k}: y_{i}=A\right\} \subset Y_{k+1} \quad \text { and } \quad\left\{i \in I \backslash T_{k}: y_{i}=B\right\} \subset Z_{k+1} .
$$


Proof of Lemma 16. a) Suppose $i \in P_{k+1}$. Then

$$
\begin{aligned}
u_{i}\left(x^{A}\right) & =u_{i}\left(x_{T_{k}}^{A}, x_{P_{k+1}}=(B, \ldots, B), x_{-T_{k}-P_{k+1}}^{A}\right) \\
& \geq u_{i}\left(x_{T_{k}}^{A}, x_{P_{k+1}}=(B, \ldots, B), x_{-T_{k}-P_{k+1}}=(\emptyset, \ldots, \emptyset)\right) \\
& >u_{i}\left(x_{T_{k}}^{A}, x_{Y_{k+1}}=(A, \ldots, A), x_{-T_{k}-Y_{k+1}}=(\emptyset, \ldots, \emptyset)\right) \\
& \geq 0
\end{aligned}
$$

where the last inequality holds trivially if $i \in\left(I \backslash T_{k}\right) \backslash Y_{k+1}$ and by the definition of $Y_{k+1}$ if $i \in Y_{k+1} \cdot u_{i}\left(x^{A}\right) \geq 0$ holds also when $i \in Q_{k+1}, R_{k+1}$ or $I \backslash T_{K}$.

b) Let $Y=\left\{i \in I \backslash T_{k}: x_{i}^{A}=A\right\}$. If $Y \not \subset Y_{k+1}$, then

$$
\begin{aligned}
& u_{i}\left(x_{T_{k}}^{A}, x_{Y \cup Y_{k+1}}=(A, \ldots, A), x_{-T_{k}-Y-Y_{k+1}}=(\emptyset, \ldots, \emptyset)\right) \\
& \geq u_{i}\left(x_{T_{k}}^{A}, x_{Y}=(A, \ldots, A), x_{-T_{k}-Y}=(\emptyset, \ldots, \emptyset)\right)=u_{i}\left(x^{A}\right) \geq 0
\end{aligned}
$$

for $i \in Y$, and

$$
\begin{aligned}
u_{i}\left(x_{T_{k}}^{A}, x_{K \cup Y_{k+1}}\right. & \left.=(A, \ldots, A), x_{-T_{k}-K-Y_{k+1}}=(\emptyset, \ldots, \emptyset)\right) \\
\geq u_{i}\left(x_{T_{k}}^{A}, x_{Y_{k+1}}\right. & \left.=(A, \ldots, A), x_{-T_{k}-Y_{k+1}}=(\emptyset, \ldots, \emptyset)\right) \geq 0
\end{aligned}
$$

for $i \in Y_{k+1}$. This contradicts the maximality of $Y_{k+1}$.

c) Let $Y=\left\{i \in J: x_{i}=A\right\}$. If $Y \not \subset Y_{k+1}$, then

$$
u_{i}\left(x_{T_{k}}^{A}, x_{Y \cup Y_{k+1}}=(A, \ldots, A), x_{-Y-Y_{k+1}}=(\emptyset, \ldots, \emptyset)\right) \geq u_{i}\left(x_{J}, x_{-J}^{A}\right) \geq 0
$$

for every $i \in Y$ because of (41), and

$$
\begin{aligned}
& u_{i}\left(x_{T_{k}}^{A}, x_{Y \cup Y_{k+1}}=(A, \ldots, A), x_{-Y-Y_{k+1}}=(\emptyset, \ldots, \emptyset)\right) \\
& \geq u_{i}\left(x_{T_{k}}^{A}, x_{Y_{k+1}}=(A, \ldots, A), x_{-T_{k}-Y_{k+1}}=(\emptyset, \ldots, \emptyset)\right) \\
& \geq 0
\end{aligned}
$$

for every $i \in Y_{k+1}$. This contradicts the maximality of $Y_{k+1}$. Hence (42) holds. (42) implies (43) if we set $J=I \backslash T_{k}$.

We now return to the proof of Proposition 14.

In what follows, denote by $J$ the deviating coalition of buyers. We first show that $x^{A}$ is a strong NE by verifying (40) for each choice of $J$ specified below. 
1) First take $J$ such that $J \cap T_{1} \neq \emptyset$.

If $J \cap P_{1} \neq \emptyset$, take $i \in J \cap P_{1}$. Then $x_{i}^{A}=B$ by the definition of $x^{A}$. If $x_{J}$ is such that $x_{i}=A$ and $u_{j}\left(x_{J}, x_{-J}^{A}\right) \geq 0$ for every $j \in J$, then

$$
\begin{aligned}
u_{i}\left(x_{J}, x_{-J}^{A}\right) & \leq u_{i}\left(x_{Y_{1}}=(A, \ldots, A), x_{-Y_{1}}=(\emptyset, \ldots, \emptyset)\right) \\
& <u_{i}\left(x_{P_{1}}=(B, \ldots, B), x_{-P_{1}}=(\emptyset, \ldots, \emptyset)\right) \\
& \leq u_{i}\left(x^{A}\right),
\end{aligned}
$$

where the first inequality follows from (41) and (42). Likewise, (40) holds for any $J$ such that $J \cap Q_{1} \neq \emptyset$. If $J$ is such that $J \cap R_{1} \neq \emptyset$, then take $i \in J \cap R_{1}$. By definition, $x_{i}^{A}=\emptyset$. If $x_{i}^{A}=A$, then since $i \notin Y_{1}$, we have $u_{i}\left(x_{J}, x_{-J}^{A}\right)<0=u_{i}\left(x^{A}\right)$. We also have $u_{i}\left(x_{J}, x_{-J}^{A}\right)<0=u_{i}\left(x^{A}\right)$ if $x_{i}=B$ since $i \notin Z_{1}$.

2) As an induction hypothesis, suppose that (40) holds for any $J$ such that $J \cap$ $T_{k} \neq \emptyset$.

Suppose that we take $J$ such that $J \cap T_{k}=\emptyset$ but $J \cap T_{k+1} \neq \emptyset$.

If $J \cap P_{k+1} \neq \emptyset$, take $i \in J \cap P_{k+1}$. Then $x_{i}^{A}=B$ by the definition of $x^{A}$. If $x_{J}$ is such that $x_{i}=A$ and $u_{j}\left(x_{J}, x_{-J}^{A}\right) \geq 0$ for every $j \in J$, then

$$
\begin{aligned}
u_{i}\left(x_{J}, x_{-J}^{A}\right) & =u_{i}\left(x_{T_{k}}^{A}, x_{J}, x_{-J-T_{k}}^{A}\right) \\
& \leq u_{i}\left(x_{T_{k}}^{A}, x_{Y_{k+1}}=(A, \ldots, A), x_{-T_{k}-Y_{k+1}}=(\emptyset, \ldots, \emptyset)\right) \\
& <u_{i}\left(x_{T_{k}}^{A}, x_{P_{k+1}}=(B, \ldots, B), x_{-T_{k}-P_{k+1}}=(\emptyset, \ldots, \emptyset)\right) \\
& \leq u_{i}\left(x^{A}\right),
\end{aligned}
$$

where the second line follows from (41) and (42). By the similar argument, (40) holds for any $J$ such that $J \cap Q_{k+1} \neq \emptyset$. If $J \cap R_{k+1} \neq \emptyset$, take $i \in J \cap R_{k+1}$. Then $x_{i}^{A}=\emptyset$ by definition. If $x_{i}=A$, then since $i \notin Y_{k+1}, u_{i}\left(x_{J}, x_{-J}^{A}\right)<0=u_{i}\left(x^{A}\right)$. We also have $u_{i}\left(x_{J}, x_{-J}^{A}\right)<u_{i}\left(x^{A}\right)$ if $x_{i}=B$ since $i \notin Z_{k+1}$.

3) Finally, suppose that $J \subset I \backslash T_{K}$. Since $P_{K+1}=\emptyset$, if $x_{J}=(B, \ldots, B)$, there exists $i \in J$ such that

$$
\begin{aligned}
u_{i}\left(x^{A}\right) & =u_{i}\left(x_{T_{K}}^{A}, x_{Y_{K+1}}=(A, \ldots, A), x_{-T_{K}-Y_{K+1}}=(\emptyset, \ldots, \emptyset)\right) \\
& \geq u_{i}\left(x_{T_{K}}^{A}, x_{J}=(B, \ldots, B), x_{-T_{K}-J}=(\emptyset, \ldots, \emptyset)\right) \\
& =u_{i}\left(x_{J}=(B, \ldots, B), x_{-J}^{A}\right),
\end{aligned}
$$


where the last equality holds because $x_{i}^{A} \neq B$ for any $i \in I \backslash T_{K}$ by definition. Clearly, no other joint deviation $x_{J}$ by $J$ yields a higher payoff for $i$ than $u_{i}\left(x_{J}=(B, \ldots, B), x_{-J}^{A}\right)$. Hence, $(40)$ holds for any $J$ such that $J \subset I \backslash T_{K}$.

We next show that the strong NE $x^{A}$ is $A$-maximal. Take any strong NE $y$. Clearly, $u_{i}(y) \geq 0$ for every $i \in I$. If $i \in P_{1}$, then $y_{i}=B$ : If $y_{i} \neq B$, then

$$
\begin{aligned}
u_{i}\left(x_{P_{1}}=(B, \ldots, B), y_{-P_{1}}\right) & >u_{i}\left(x_{Y_{1}}=(A, \ldots, A), x_{-Y_{1}}=(\emptyset, \ldots, \emptyset)\right) \\
& \geq u_{i}(y),
\end{aligned}
$$

where the last inequality from (43) for $k=0$. Hence, $y$ violates (40). Likewise, we can conclude that

$$
y_{i}= \begin{cases}A & \text { if } i \in Q_{1}, \\ \emptyset & \text { if } i \in R_{1} .\end{cases}
$$

Hence, $y_{T_{1}}=x_{T_{1}}^{A}$. As an induction hypothesis, suppose that $y_{T_{k}}=x_{T_{k}}^{A}$. If $i \in P_{k+1}$ then $y_{i}=B$ : If $y_{i} \neq B$, then

$$
\begin{aligned}
& u_{i}\left(y_{T_{k}}, x_{P_{k+1}}=(B, \ldots, B), y_{-T_{k}-P_{k+1}}\right) \\
& >u_{i}\left(y_{T_{k}}, x_{Y_{k+1}}=(A, \ldots, A), x_{-T_{k}-Y_{k+1}}=(\emptyset, \ldots, \emptyset)\right) \\
& \geq u_{i}\left(y_{T_{k}}, y_{-T_{k}}\right)
\end{aligned}
$$

where the last inequality follows from (43) since $y_{T_{k}}=x_{T_{k}}^{A}$ by the induction hypothesis. Hence, $y$ violates (40). We also have

$$
y_{i}= \begin{cases}A & \text { if } i \in Q_{k+1} \\ \emptyset & \text { if } i \in R_{k+1}\end{cases}
$$

Hence, $y_{T_{k+1}}=x_{T_{k+1}}^{A}$. Suppose finally that $i \in I \backslash T_{K}$. Since $\left\{i \in I \backslash T_{K}:, y_{i}=\right.$ $A\} \subset Y_{K+1}$ by (43), $y_{i}=A$ implies $x_{i}^{A}=A$, showing that $x^{A}$ is $A$-maximal.

Proof of Proposition 15. In view of Proposition 9, it suffices to check the existence of a strong NE when $G$ is either cyclic or complete.

1) $G$ is a cycle.

Suppose that for $\varepsilon>0$ small, $p$ is given by

$$
p_{i}= \begin{cases}v^{1}-v^{2}-\varepsilon & \text { if } i=1, N-1, \\ -\varepsilon & \text { if } i=2, \ldots, N-2, \\ v^{2}-v^{0}-\varepsilon & \text { if } i=N\end{cases}
$$


We can then verify that under $\left(p, q^{*}\right), Q_{1}=\{1, \ldots, N-1\}$ : For $i=1$ and $N-1$,

$$
\begin{aligned}
u_{i}\left(x_{Q_{1}}=(A, \ldots, A), x_{N}=\emptyset\right) & =v^{1}-p_{i} \\
& =v^{2}+\varepsilon \\
& >u_{i}(x=(B, \ldots, B)),
\end{aligned}
$$

and for $i=2, \ldots, N-2$,

$$
\begin{aligned}
u_{i}\left(x_{Q_{1}}=(A, \ldots, A), x_{N}=\emptyset\right) & =v^{2}-p_{i} \\
& =v^{2}+\varepsilon \\
& >u_{i}(x=(B, \ldots, B)),
\end{aligned}
$$

but for $i=N$,

$$
\begin{aligned}
u_{N}(x=(A, \ldots, A)) & =v^{2}-p_{N} \\
& =v^{0}+\varepsilon \\
& <u_{N}(x=(B, \ldots, B)) .
\end{aligned}
$$

We can also verify that $P_{1}=R_{1}=\emptyset$. Given $T_{1}=I \backslash\{N\}, Q_{2}=\{N\}$ :

$$
u_{N}\left(x_{T_{1}}, x_{N}=A\right)=v^{2}-p_{N}=v^{0}+\varepsilon>u_{N}\left(x_{T_{1}}, x_{N}=B\right) .
$$

Therefore, $Q_{1} \cup Q_{2}=I$ and seller $A$ 's payoff under $\left(p, q^{*}\right)$ equals

$$
\pi_{A}\left(p, q^{*}, \sigma\right)=2\left(v^{1}-v^{2}-\varepsilon\right)+v^{2}-v^{0}-\varepsilon=2 v^{1}-v^{0}-v^{2}-3 \varepsilon,
$$

which is strictly positive if $2 v^{1}-v^{0}-v^{2}>0$ and $\varepsilon$ is sufficiently small. If $2 v^{1}-v^{0}-$ $v^{2}<0$, then we can verify that $\pi_{A}\left(p, q^{*}, \sigma\right)>0$ if we take $\varepsilon>0$ small and $p$ such that

$$
p_{i}= \begin{cases}v^{2}-v^{1}-\varepsilon & \text { if } i=1, N-1, \\ -\varepsilon & \text { if } i=2, \ldots, N-2, \\ v^{0}-v^{2}-\varepsilon & \text { if } i=N\end{cases}
$$

2) Suppose next that $G$ is complete.

Consider $p$ such that

$$
p_{i}= \begin{cases}v^{N-2}-v^{N-1}-\varepsilon & \text { if } i=1, \ldots, N-1, \\ v^{N-1}-v^{0}-\varepsilon & \text { if } i=N .\end{cases}
$$


Then $Q_{1}=\{1, \ldots, N-1\}$ and $Q_{2}=\{N\}$, and

$$
\begin{aligned}
\pi_{A}\left(p, q^{*}, \sigma\right) & =(N-1)\left(v^{N-2}-v^{N-1}-\varepsilon\right)+v^{N-1}-v^{0}-\varepsilon \\
& =(N-1) v^{N-2}-(N-2) v^{N-1}-v^{0}-N \varepsilon,
\end{aligned}
$$

which is strictly positive if $(N-1) v^{N-2}-(N-2) v^{N-1}-v^{0}>0$ and $\varepsilon$ is sufficiently small. If $(N-1) v^{N-2}-(N-2) v^{N-1}-v^{0}<0$, then $\pi_{A}\left(p, q^{*}, \sigma\right)>0$ if $\varepsilon>0$ is small and $p$ is given by

$$
p_{i}= \begin{cases}v^{N-1}-v^{N-2}-\varepsilon & \text { if } i=1, \ldots, N-1, \\ v^{0}-v^{N-1}-\varepsilon & \text { if } i=N .\end{cases}
$$

In either case, hence, seller $A$ can profitably deviate from $p^{*}=0$ against $q^{*}=0$.

\section{References}

[1] Attila Ambrus, and Rossela Argenziano (2009), "Asymmetric networks in twosided markets," American Economic Journal: Microeconomics, 1(1), 17-52.

[2] Masaki Aoyagi (2013), "Coordinating adoption decisions under externalities and incomplete information," Games and Economic Behavior, 77, 77-89.

[3] Mark Armstrong (1998), "Network interconnection in telecommunications," Economic Journal, 108, 545-564.

[4] A. Banerji, and Bhaskar Dutta (2009), "Local network externalities and market segmentation," International Journal of Industrial Organization, 27, 605-614.

[5] Douglas B. Bernheim, Bezalel Peleg, and Michael D. Whinston (1987), "Coalition-proof Nash equilibria: concepts," Journal of Economic Theory, 42(1), 1-12.

[6] Shai Bernstein, and Eyal Winter (2012), "Contracting with heterogeneous externalities," American Economic Journal: Microeconomics, 4(2), 50-76.

[7] Luis M. B. Cabral, David J. Salant, and Glenn A. Woroch (1999), "Monopoly pricing with network externalities," International Journal of Industrial Organization, 17, 199-214.

[8] Philip H. Dybvig, and Chester S. Spatt (1983), "Adoption externalities as public goods," Journal of Public Economics, 20, 231-247. 
[9] Bruno Jullien (2011), "Competition in multi-sided markets: divide and conquer," American Economic Journal: Microeconomics, 3, 186-219.

[10] Michael L. Katz, and Carl Shapiro (1985), "Network externalities, competition, and compatibility," American Economic Review, 75, 424-440.

[11] Jean-Jacques Laffont, Patrick Rey, and Jean Tirole (1998), "Network competition: I. Overview and nondiscriminatory pricing," Rand Journal of Economics, 29(1) $1-37$.

[12] Jean-Jacques Laffont, Patrick Rey, and Jean Tirole (1998), "Network competition: II. Price Discrimination," Rand Journal of Economics, 29(1) 38-56.

[13] Jack Ochs, and In-Uck Park (2010), "Overcoming the coordination problem: dynamic formation of networks," Journal of Economic Theory, 145, 689-720.

[14] In-Uck Park (2004), "A simple inducement scheme to overcome adoption externalities," Contributions to Theoretical Economics, 4(1), Article 3.

[15] Jeffrey H. Rohlfs (1974), "A theory of interdependent demand for a communications service," Bell Journal of Economics, 5 (1), 16-37.

[16] Ilya Segal (2003), "Coordination and discrimination in contracting with externalities: Divide and conquer?" Journal of Economic Theory, 113, 147-327.

[17] Tadashi Sekiguchi (2009), "Pricing of durable network goods under dynamic coordination failure," working paper.

[18] Arun Sundararajan (2003), "Network effects, nonlinear pricing and entry deterrence," discussion paper, NYU. 